\title{
A generalized isospectral-nonisospectral heat equation hierarchy and its expanding integrable model
}

\author{
Huanhuan Lu', Yufeng Zhang ${ }^{1 *}$ and Jianqin $\mathrm{Mei}^{2}$
}

\section{"Correspondence:}

zyfxz@cumt.edu.cn

'School of Mathematics, China

University of Mining and

Technology, Xuzhou, Jiangsu

221116, China

Full list of author information is

available at the end of the article

\begin{abstract}
A generalized nonisospectral heat integrable hierarchy with three dependent variables is singled out. A Bäcklund transformation of a resulting isospectral integrable hierarchy is produced by converting the usual Lax pair into the Lax pairs in Riccati forms. In addition, an expanding integrable model is also worked out by making use of a set of linear spectral problems which are introduced via a high-dimensional loop algebra. Finally, we obtain some conserved densities of a types of heat integrable system by using the weight theory on PDEs.
\end{abstract}

Keywords: Nonisospectral integrable hierarchy; Bäcklund transformation; Conserved density

\section{Introduction}

There has been an important aspect of the search for integrable systems and their algebraic and geometric properties in soliton theory. Magri [1] proposed the Lax pair method to generate integrable equations. Ablowitz [2,3], and Newell et al. [4] have made great contributions on integrable systems, including the methods for generating integrable equations. Tu Guizhang [5] once applied various loop algebras to introduce linear isospectral problems in order to efficiently generate new integrable hierarchies of evolution equations and the corresponding Hamiltonian structures. Ma Wen-xiu [6] called the approach the Tu scheme. By applying the Tu scheme, many interesting integrable systems and their properties were obtained, such as the work in [7-15]. Guo Fukui [16] once applied the following loop algebra:

$$
\begin{aligned}
& \widetilde{A_{1}}=\operatorname{span}\{h(n), e(n), f(n)\}, \\
& h(n)=\frac{1}{2}\left(\begin{array}{cc}
\lambda^{2 n+1} & 0 \\
0 & -\lambda^{2 n+1}
\end{array}\right), \quad e(n)=\frac{1}{2}\left(\begin{array}{cc}
0 & \lambda^{2 n+1} \\
\lambda^{2 n+1} & 0
\end{array}\right), \\
& f(n)=\frac{1}{2}\left(\begin{array}{cc}
0 & \lambda^{2 n} \\
-\lambda^{2 n} & 0
\end{array}\right),
\end{aligned}
$$

(c) The Author(s) 2020. This article is licensed under a Creative Commons Attribution 4.0 International License, which permits use, sharing, adaptation, distribution and reproduction in any medium or format, as long as you give appropriate credit to the original author(s) and the source, provide a link to the Creative Commons licence, and indicate if changes were made. The images or other third party material in this article are included in the article's Creative Commons licence, unless indicated otherwise in a credit line to the material. If material is not included in the article's Creative Commons licence and your intended use is not permitted by statutory regulation or exceeds the permitted use, you will need to obtain permission directly from the copyright holder. To view a copy of this licence, visit http://creativecommons.org/licenses/by/4.0/. 
along with the commutators

$$
\begin{aligned}
& {[h(n), e(n)]=f(m+n+1), \quad[h(m), f(n)]=e(m+n),} \\
& {[f(m), e(n)]=h(m+n), \quad m, n \in Z,}
\end{aligned}
$$

to introduce the following isospectral problems:

$$
\begin{aligned}
& \varphi_{x}=U \varphi, \quad \lambda_{t}=0, \quad U=f(1)+q e(0)+\operatorname{rh}(0), \\
& \varphi_{t}=V^{(n)} \varphi, \quad V^{(n)}=\sum_{m=0}^{n}\left(a_{m} f(n-m)+b_{m} e(n-m)+c_{m} h(n-m)\right)-c_{n} f(0),
\end{aligned}
$$

whose compatibility condition yields the isospectral integrable hierarchy

$$
\begin{aligned}
& \left(\begin{array}{l}
q \\
r
\end{array}\right)_{t}=\left(\begin{array}{c}
-a_{n+1}+r c_{n} \\
b_{n+1}-q c_{n}
\end{array}\right)=\left(\begin{array}{l}
b_{n x} \\
a_{n x}
\end{array}\right)=\left(\begin{array}{ll}
\partial & 0 \\
0 & \partial
\end{array}\right)\left(\begin{array}{l}
b_{n} \\
c_{n}
\end{array}\right)=: J\left(\begin{array}{l}
b_{n} \\
c_{n}
\end{array}\right), \\
& \left(\begin{array}{l}
b_{n+1} \\
c_{n+1}
\end{array}\right)=L\left(\begin{array}{l}
b_{n} \\
c_{n}
\end{array}\right), \quad L=\left(\begin{array}{cc}
q \partial^{-1} q \partial & \partial+q \partial^{-1} r \partial \\
-\partial+r \partial^{-1} q \partial & r \partial^{-1} r \partial
\end{array}\right),
\end{aligned}
$$

where $J=\left(\begin{array}{ll}\partial & 0 \\ 0 & \partial\end{array}\right), L$ is called a recurrence operator.

As we have known that schemes for generating nonisospectral integrable hierarchies of evolution equations are less than those for isospectral integrable hierarchies. Ma $[17,18]$ made use of Lax operators and zero curvature equations to investigate the nonisospectral integrable equations and their algebraic structures. Qiao Zhijun $[19,20]$ adopted the generalized Lax representations to generate nonisospectral integrable hierarchies and further discuss their algebraic structures. We find that all of the nonisospectral integrable hierarchies were obtained under the assumption $\lambda_{t}=\lambda^{n}(n \geq 0)$. However, Li Yishen, [21] utilized the zero curvature equation and the time evolution $\lambda_{t}=\sum_{j=0}^{n} k_{j}(t) \lambda^{n-j}$ to work out some nonisospectral integrable hierarchies. Li and Zhuang [22] made use of the time evolution $\lambda_{t}=\sum_{j=0}^{m-1} k_{2 j+1}(t) \lambda^{2(m-j)-1}$ and a nonisospectral zero curvature equation to produce the nonisospectral WKI integrable hierarchy of evolution equations, but it is difficult to understand the paper. In order to straightforward generate nonisospectral hierarchies of evolution equations. Zhang, et al. [23] proposed a method which applies the Tu scheme and Li's method [21,22] to derive nonisospectral integrable hierarchies and investigate the symmetries of integrable hierarchies. In this paper, we would like to adopt the method to work out the nonisospectral integrable hierarchies corresponding to the Guo hierarchy [3]. Through enlarging the loop algebra $A_{1}$ as above [24]. we obtain an expanding isospectral-nonisospectral integrable hierarchy which reduces to the Guo hierarchy. Specially, we further study the Bäcklund transformation of the isospectral integrable hierarchy [3]. Finally, we reduce the isospectral-nonisospectral integrable hierarchy to a type of heat equation which can be reduced to the standard linear equation, whose format of conserved densities is singled out.

\section{An isospectral-nonisospectral integrable hierarchy and its reduction}

We take

$$
V=V_{1}+V_{2}, \quad V_{1}=\sum_{i \geq 0}\left(a_{i} h(-i)+b_{i} e(-i)+c_{i} f(-i)\right)
$$




$$
\begin{aligned}
& V_{2}=\sum_{j \geq 0}\left(\bar{a}_{j} h(-j)+\bar{b}_{j} e(-j)+\bar{c}_{j} f(-j)\right), \\
& \lambda_{t}=\frac{\partial \lambda}{\partial t}=\sum_{j \geq 0} k_{j}(t) \lambda^{1-2 j} .
\end{aligned}
$$

First solving the stationary zero curvature equation,

$$
V_{1, x}=\left[U, V_{1}\right]
$$

gives rise to

$$
\left\{\begin{array}{l}
a_{i x}=b_{i+1}-q c_{i}, \\
a_{i+1}=-b_{i x}+r c_{i}, \\
c_{i x}=-q a_{i+1}+r b_{i+1}=r a_{i x}+q b_{i x}, \quad i \geq 0
\end{array}\right.
$$

from which we have $c_{i}=\partial^{-1}\left(r a_{i x}+q b_{i x}\right)+\beta_{i}(t)$. According to the work in [16], then the compatibility condition of Eq. (1) and Eq. (2), that is, the zero curvature equation

$$
U_{t}-V_{1, x}^{(n)}+\left[U, V_{1}^{(n)}\right]=0
$$

admits the Guo hierarchy Eq. (3).

However, under the time evolution $\lambda_{t} \neq 0$, the resulting zero curvature equation differs from Eq. (6). We first solve the following evolution equation in terms of the Tu scheme and the method presented in Eq. (23):

$$
V_{2, x}=\left[U, V_{2}\right]+\frac{\partial U}{\partial \lambda} \lambda_{t},
$$

which leads to

$$
\left\{\begin{array}{l}
\bar{a}_{j x}=\bar{b}_{j+1}-q \bar{c}_{j}+k_{j}(t) r, \\
\bar{b}_{j x}=-\bar{a}_{j+1}+r \bar{c}_{j}+k_{j}(t) q, \\
\bar{c}_{j x}=-q \bar{a}_{j+1}+r \bar{b}_{j+1}+2 k_{j+1}(t), \quad j \geq 0,
\end{array}\right.
$$

from which we have

$$
\begin{aligned}
\bar{c}_{j x} & =q \bar{b}_{j x}+r \bar{a}_{j x}-k_{j}(t)\left(q^{2}+r^{2}\right)+2 k_{j+1}(t) \\
& \Rightarrow \quad \bar{c}_{j}=\partial^{-1}\left(q \bar{b}_{j x}+r \bar{a}_{j x}\right)-k_{j}(t) \partial^{-1}\left(q^{2}+r^{2}\right)+2 k_{j+1}(t) x .
\end{aligned}
$$

Denoting

$$
\begin{aligned}
& V_{2,+}^{(m)}=\sum_{j=0}^{m}\left(\bar{a}_{j} h(m-j)+\bar{b}_{j} e(m-j)+\bar{c}_{j} f(m-j)\right)=\lambda^{2 m} V_{2}-V_{2,-}^{(m)}, \\
& \lambda_{t,+}^{(m)}=\sum_{j=0}^{m} k_{j}(t) \lambda^{2 m-2 j+1}=\lambda^{2 m} \lambda_{t}-\lambda_{t,-}^{(m)}=\lambda^{2 m} \lambda_{t}-\sum_{j=m+1}^{\infty} k_{j}(t) \lambda^{2 m-2 j+1},
\end{aligned}
$$


then Eq. (7) can be decomposed into the following form:

$$
-\left(V_{2,+}^{(m)}\right)_{x}+\left[U, V_{2,+}^{(m)}\right]+\frac{\partial U}{\partial \lambda} \lambda_{t,+}^{(m)}=\left(V_{2,-}^{(m)}\right)_{x}-\left[U, V_{2,-}^{(m)}\right]-\frac{\partial U}{\partial \lambda} \lambda_{t,-}^{(m)} .
$$

The gradations of the left-hand side in Eq. (9) are more than 0, while those of the righthand side are less than 1 . Hence, we only take the terms with the gradations being 0 and 1 in both sides of Eq. (9), and

$$
-\left(V_{2,+}^{(m)}\right)_{x}+\left[U, V_{2,+}^{(m)}\right]+\frac{\partial U}{\partial \lambda} \lambda_{t,+}^{(m)}=\bar{a}_{m+1} e(0)-\bar{b}_{m+1} h(0)-\bar{c}_{m x} f(0) .
$$

Let $V_{2}^{(m)}=V_{2,+}^{(m)}-\bar{c}_{m} f(0)$, a direct calculation shows that

$$
-\left(V_{2}^{(m)}\right)_{x}+\left[U, V_{2}^{(m)}\right]+\frac{\partial U}{\partial \lambda} \lambda_{t,+}^{(m)}=\left(\bar{a}_{m+1}-r \bar{c}_{m}\right) e(0)+\left(-\bar{b}_{m+1}+q \bar{c}_{m}\right) h(0) .
$$

Thus, the nonisospectral zero curvature equation,

$$
U_{t}-V_{1, x}^{(n)}-V_{2, x}^{(m)}+\left[U, V_{1}^{(n)}+V_{2}^{(m)}\right]=0,
$$

gives an isospectral-nonisospectral integrable hierarchy,

$$
\begin{aligned}
\left(\begin{array}{l}
q \\
t
\end{array}\right)_{t_{n, m}} & =\left(\begin{array}{l}
-a_{n+1}+r c_{n}-\bar{a}_{m+1}+r \bar{c}_{m} \\
b_{n+1}-q c_{n}+\bar{b}_{m+1}-q \bar{c}_{m}
\end{array}\right)=\left(\begin{array}{l}
b_{n x}+\bar{b}_{m x}-k_{m}(t) q \\
a_{n x}+\bar{a}_{m x}-k_{m}(t) r
\end{array}\right) \\
& =\left(\begin{array}{ll}
\partial & 0 \\
0 & \partial
\end{array}\right)\left(\begin{array}{l}
b_{n}+\bar{b}_{m} \\
a_{n}+\bar{a}_{m}
\end{array}\right)-k_{m}(t)\left(\begin{array}{l}
q \\
r
\end{array}\right) \\
& =J\left(\begin{array}{l}
b_{n} \\
a_{n}
\end{array}\right)+J\left(\begin{array}{l}
\bar{b}_{m} \\
\bar{a}_{m}
\end{array}\right)-k_{m}(t)\left(\begin{array}{l}
q \\
r
\end{array}\right) .
\end{aligned}
$$

In what follows, we consider some reductions for Eq. (11). Let $a_{0}=b_{0}=0, c_{0}=\beta_{0}(t), \bar{a}_{0}=$ $\bar{b}_{0}=0$, then we have from Eq. (5) and Eq. (8)

$$
\begin{aligned}
a_{1}= & \beta_{0}(t) r, \quad b_{1}=\beta_{0}(t) q, \\
c_{1}= & \frac{1}{2} \beta_{0}(t)\left(q^{2}+r^{2}\right)+\beta_{1}(t), \\
\bar{a}_{1}= & -k_{0}(t) r \partial^{-1}\left(q^{2}+r^{2}\right)+k_{0}(t) q+2 k_{1}(t) x r, \\
\bar{b}_{1}= & -k_{0}(t) q \partial^{-1}\left(q^{2}+r^{2}\right)-k_{0}(t) r+2 k_{1}(t) x q, \\
\bar{c}_{0}= & -k_{0}(t) \partial^{-1}\left(q^{2}+r^{2}\right)+2 k_{1}(t) x, \\
\bar{c}_{1}= & -\frac{1}{2} k_{0}(t)\left(q^{2}+r^{2}\right) \partial^{-1}\left(q^{2}+r^{2}\right)-\frac{1}{2} k_{0}(t) \partial^{-1}\left(q^{2}+r^{2}\right)^{2}+k_{0}(t) \partial^{-1}\left(q_{x} r-q r_{x}\right) \\
& -k_{1}(t) \partial^{-1}\left(q^{2}+r^{2}\right)+2 k_{2}(t) x, \\
a_{2}= & -\beta_{0}(t) q_{x}+\frac{1}{2} \beta_{0}(t) r\left(q^{2}+r^{2}\right)+\beta_{1}(t) r, \\
b_{2}= & \beta_{0}(t) r_{x}+\frac{1}{2} \beta_{0}(t) q\left(q^{2}+r^{2}\right)+\beta_{1}(t) q,
\end{aligned}
$$




$$
\begin{aligned}
\bar{a}_{2}= & k_{0}(t) q_{x} \partial^{-1}\left(q^{2}+r^{2}\right)+k_{0}(t) q\left(q^{2}+r^{2}\right)+k_{0}(t) r_{x} \\
& -\frac{1}{2} k_{0}(t) r\left(q^{2}+r^{2}\right) \partial^{-1}\left(q^{2}+r^{2}\right)-\frac{1}{2} k_{0}(t) r \partial^{-1}\left(q^{2}+r^{2}\right)^{2} \\
& +k_{0}(t) r \partial^{-1}\left(q_{x} r-q r_{x}\right)-k_{1}(t) r \partial^{-1}\left(q^{2}+r^{2}\right)+2 k_{1}(t) q+2 k_{2}(t) x r, \\
\bar{b}_{2}= & -k_{0}(t) r_{x} \partial^{-1}\left(q^{2}+r^{2}\right)-k_{0}(t) r\left(q^{2}+r^{2}\right)+k_{0}(t) q_{x}-\frac{1}{2} k_{0}(t) q\left(q^{2}+r^{2}\right) \partial^{-1}\left(q^{2}+r^{2}\right) \\
& -\frac{1}{2} k_{0}(t) q \partial^{-1}\left(q^{2}+r^{2}\right)^{2}+k_{0}(t) q \partial^{-1}\left(q_{x} r-q r_{x}\right)-k_{1}(t) q \partial^{-1}\left(q^{2}+r^{2}\right) \\
& -k_{1}(t) r-2 k_{2}(t) x q,
\end{aligned}
$$

Hence, we see that

$$
\left(\begin{array}{l}
q \\
r
\end{array}\right)_{t_{0,1}}=\left(\begin{array}{l}
\bar{b}_{1 x}-k_{1}(t) q \\
\bar{a}_{1 x}-k_{1}(t) r
\end{array}\right)=-k_{0}(t)\left(\begin{array}{l}
\left(q \partial^{-1}\left(q^{2}+r^{2}\right)\right)_{x}-r_{x} \\
\left(r \partial^{-1}\left(q^{2}+r^{2}\right)\right)_{x}+q_{x}
\end{array}\right)+2 k_{1}(t)\left(\begin{array}{c}
(x q)_{x} \\
(x r)_{x}
\end{array}\right) .
$$

Taking $q^{2}+r^{2}=\rho_{x}$, then Eq. (12) becomes

$$
\left\{\begin{array}{l}
q_{t}=-k_{0}(t)(q \rho)_{x}+k_{0}(t) r_{x}-2 k_{1}(t)(x q)_{x} \\
r_{t}=-k_{0}(t)(r \rho)_{x}-k_{0}(t) q_{x}+2 k_{1}(t)(x r)_{x}
\end{array}\right.
$$

which can be written, when $k_{0}(t)=1$,

$$
\left\{\begin{array}{l}
q_{t}+\rho q_{x}=-q \rho_{x}+r_{x}-2 k_{1}(t)(x q)_{x} \\
r_{t}+\rho r_{x}=-r \rho_{x}-q_{x}+2 k_{1}(t)(x r)_{x} \\
\rho_{x}=q^{2}+r^{2}
\end{array}\right.
$$

Remark 1 This nonlocal integrable system is obviously an extension of the nonlocal integrable system given by $\mathrm{Hu}$ and $\mathrm{Li}$ [25],

$$
\left\{\begin{array}{l}
m_{t}+\rho m_{x}=-m(\psi(t, x)-\bar{\psi}(t)), \\
n_{t}+\rho n_{x}=-n(\psi(t, x)-\bar{\psi}(t)), \\
\rho=\partial_{x}^{-1} \psi
\end{array}\right.
$$

Zhang and Qiao [26] investigated the periodic Cauchy problem and the well-posedness as well as the blow-up phenomena of Eq. (14). Therefore, there is an open problem asking whether we could study the Cauchy problem, the blow-up phenomena of the system of equations (13), which will be discussed in another paper in the forthcoming days.

In fact, we can get other reduced integrable systems from Eq. (11). For example, we have

$$
\begin{aligned}
& \left(\begin{array}{l}
q \\
r
\end{array}\right)_{t_{1,1}} \\
& =\left(\begin{array}{c}
\beta_{0}(t) q_{x}-k_{0}(t) q_{x} \partial^{-1}\left(q^{2}+r^{2}\right)-k_{0}(t) q\left(q^{2}+r^{2}\right)-k_{0}(t) r_{x}+k_{1}(t) q+2 k_{1}(t) x q_{x x} \\
\beta_{0}(t) r_{x}-k_{0}(t) r_{x} \partial^{-1}\left(q^{2}+r^{2}\right)-k_{0}(t) r\left(q^{2}+r^{2}\right)+k_{0}(t) q_{x}+k_{1}(t) r+2 k_{1}(t) x r_{x x}
\end{array}\right),
\end{aligned}
$$




$$
\left(\begin{array}{l}
q \\
r
\end{array}\right)_{t_{2,0}}=\left(\begin{array}{c}
\beta_{0}(t) r_{x x}+\frac{1}{2} \beta_{0}(t)\left(q\left(q^{2}+r^{2}\right)\right)_{x}-k_{0}(t) q \\
-\beta_{0}(t) q_{x x}+\frac{1}{2} \beta_{0}(t)\left(r\left(q^{2}+r^{2}\right)\right)_{x}-k_{0}(t) r
\end{array}\right)
$$

Taking $q=i r$, Eq. (16) reduces to

$$
q_{t}=i \beta_{0}(t) q_{x x}-k_{0}(t) q
$$

Specially, set $i \beta_{0}(t)>0, k_{0}(t)=0$, Eq. (17) becomes to the standard linear heat equation

$$
q_{t}=\alpha q_{x x}, \quad \alpha>0 .
$$

Hence, we call the following reduced integrable system from Eq. (16) a type of heat equation:

$$
\begin{aligned}
& \left\{\begin{array}{l}
q_{t}=2 r_{x x}+\left(q\left(q^{2}+r^{2}\right)\right)_{x}, \\
r_{t}=-2 q_{x x}+\left(r\left(q^{2}+r^{2}\right)\right)_{x},
\end{array}\right. \\
& q_{t_{1,2}}=\beta_{0}(t) q_{x}-k_{0}(t) r_{x x} \partial^{-1}\left(q^{2}+r^{2}\right)-k_{0}(t) r_{x}\left(q^{2}+r^{2}\right)-k_{0}(t) r\left(q^{2}+r^{2}\right)_{x} \\
& +k_{0}(t) q_{x x}-\frac{1}{2} k_{0}(t)\left(q\left(q^{2}+r^{2}\right)\right)_{x} \partial^{-1}\left(q^{2}+r^{2}\right)-k_{0}(t) q\left(q^{2}+r^{2}\right)^{2} \\
& -\frac{1}{2} k_{0}(t) q_{x} \partial^{-1}\left(q^{2}+r^{2}\right)^{2}+k_{0}(t) q_{x} \partial^{-1}\left(q_{x} r-q r_{x}\right)+k_{0}(t) q\left(q_{x} r-q r_{x}\right) \\
& -k_{1}(t) q_{x} \partial^{-1}\left(q^{2}+r^{2}\right)-k_{1}(t) q\left(q^{2}+r^{2}\right)_{x}+k_{2}(t) q+2 k_{2}(t) x q_{x}-k_{1}(t) r_{x}, \\
& r_{t_{1,2}}=\beta_{0}(t) r_{x}+k_{0}(t) q_{x x} \partial^{-1}\left(q^{2}+r^{2}\right)+k_{0}(t) q_{x}\left(q^{2}+r^{2}\right)+k_{0}(t) q\left(q^{2}+r^{2}\right)_{x} \\
& +k_{0}(t) r_{x x}-\frac{1}{2} k_{0}(t)\left(r\left(q^{2}+r^{2}\right)\right)_{x} \partial^{-1}\left(q^{2}+r^{2}\right)-k_{0}(t) r\left(q^{2}+r^{2}\right)^{2} \\
& -\frac{1}{2} k_{0}(t) r_{x} \partial^{-1}\left(q^{2}+r^{2}\right)+k_{0}(t) r_{x} \partial^{-1}\left(q_{x} r-q r_{x}\right)+k_{0}(t) r\left(q_{x} r-q r_{x}\right) \\
& -k_{1}(t) r_{x} \partial^{-1}\left(q^{2}+r^{2}\right)-k_{1}(t) r\left(q^{2}+r^{2}\right)_{x}+k_{2}(t) r+2 k_{2}(t) x r_{x}-k_{1}(t) q_{x},
\end{aligned}
$$

set $k_{0}(t)=\beta_{0}(t)=0$, Eq. (19) and Eq. (20) reduce to

$$
\left\{\begin{array}{l}
q_{t}=-k_{1}(t) q_{x} \partial^{-1}\left(q^{2}+r^{2}\right)-k_{1}(t) q\left(q^{2}+r^{2}\right)_{x}+k_{2}(t) q+2 k_{2}(t) x q_{x}-k_{1}(t) r_{x}, \\
r_{t}=-k_{1}(t) r_{x} \partial^{-1}\left(q^{2}+r^{2}\right)-k_{1}(t) r\left(q^{2}+r^{2}\right)_{x}+k_{2}(t) r+2 k_{2}(t) x r_{x}+k_{1}(t) q_{x} .
\end{array}\right.
$$

Let $k_{1}=0$, Eq. (21) further reduces to a variable coefficient linear equation

$$
q_{t}=k_{2}(t)\left(q+2 x q_{x}\right)
$$

Assume $k_{2}=0$, Eq. (21) becomes

$$
\left\{\begin{array}{l}
q_{t}=-k_{1}(t)\left[q_{x} \partial^{-1}\left(q^{2}+r^{2}\right)+q\left(q^{2}+r^{2}\right)_{x}+r_{x}\right] \\
r_{t}=-k_{1}(t)\left[r_{x} \partial^{-1}\left(q^{2}+r^{2}\right)+r\left(q^{2}+r^{2}\right)_{x}-q_{x}\right]
\end{array}\right.
$$

Let $q^{2}+r^{2}=\rho_{x}, k_{1}=-1$, Eq. (23) is just right Eq. (13). 
From the above discussions, we can get many nonlocal integrable systems from the integrable hierarchy Eq. (13). Hence, Eq. (13) is a source to generate nonlocal integrable systems. Based on this, we could follow the method presented in $[27,28]$ to investigate their algebraic and geometric properties. In what follows, we consider the recurrence operators of the nonisospectral integrable hierarchy Eq. (11). since we have

$$
\left(\begin{array}{c}
b_{i+1} \\
a_{i+1}
\end{array}\right)=L\left(\begin{array}{l}
b_{i} \\
a_{i}
\end{array}\right)+\beta_{i}(t)\left(\begin{array}{l}
q \\
r
\end{array}\right),
$$

via the induction method we get

$$
\left(\begin{array}{l}
b_{i} \\
a_{i}
\end{array}\right)=\left(\beta_{0} L^{i-1}+\beta_{1} L^{i-2}+\cdots+\beta_{i-1}\right)\left(\begin{array}{l}
q \\
r
\end{array}\right), \quad i=1,2, \ldots, n .
$$

In terms of Eq. (8), one has

$$
\begin{gathered}
\bar{b}_{j+1}=\bar{a}_{j x}+q\left[\partial^{-1}\left(q \bar{b}_{j x}+r \bar{a}_{j x}\right)-k_{j}(t) \partial^{-1}\left(q^{2}+r^{2}\right)+2 k_{j+1}(t) x\right]-k_{j}(t) r, \\
\bar{a}_{j+1}=-\bar{b}_{j x}+r\left[\partial^{-1}\left(q \bar{b}_{j x}+r \bar{a}_{j x}\right)-k_{j}(t) \partial^{-1}\left(q^{2}+r^{2}\right)+2 k_{j+1}(t) x\right]+k_{j}(t) q, \\
\left(\begin{array}{l}
\bar{b}_{j+1} \\
\bar{a}_{j+1}
\end{array}\right)=\left(\begin{array}{cc}
q \partial^{-1} q \partial & \partial+q \partial^{-1} r \partial \\
-\partial+r \partial^{-1} q \partial & r \partial^{-1} r \partial
\end{array}\right)\left(\begin{array}{l}
\bar{b}_{j} \\
\bar{a}_{j}
\end{array}\right) \\
+\left(\begin{array}{c}
-k_{j}(t) q \partial^{-1}\left(q^{2}+r^{2}\right)-k_{j}(t) r+2 k_{j}(t) x q \\
-k_{j}(t) r \partial^{-1}\left(q^{2}+r^{2}\right)+k_{j}(t) q+2 k_{j+1}(t) x r
\end{array}\right) \\
=: L\left(\begin{array}{c}
\bar{b}_{j} \\
\bar{a}_{j}
\end{array}\right)+k_{j}(t)\left(\begin{array}{l}
-q \partial^{-1}\left(q^{2}+r^{2}\right)-r \\
-r \partial^{-1}\left(q^{2}+r^{2}\right)+q
\end{array}\right)+2 k_{j+1}(t)\left(\begin{array}{c}
x q \\
x r
\end{array}\right) .
\end{gathered}
$$

Therefore,

$$
\begin{aligned}
\left(\begin{array}{c}
\bar{b}_{j} \\
\bar{a}_{j}
\end{array}\right)= & \left(k_{0}(t) L^{j-1}+k_{1}(t) L^{j-2}+\cdots+k_{j-1}(t)\right)\left(\begin{array}{c}
-q \partial^{-1}\left(q^{2}+r^{2}\right)-r \\
-r \partial^{-1}\left(q^{2}+r^{2}\right)+q
\end{array}\right) \\
& +2\left(k_{1}(t) L^{j}+k_{2}(t) L^{j-1}+\cdots+k_{j}(t) L\right)\left(\begin{array}{c}
x q \\
x r
\end{array}\right) .
\end{aligned}
$$

Thus, the integrable hierarchy Eq. (11) can be written as

$$
\begin{aligned}
\left(\begin{array}{l}
q \\
r
\end{array}\right)_{t_{n, m}}= & \sum_{j=0}^{n-1} \beta_{j}(t) J L^{n-1-j}\left(\begin{array}{l}
q \\
r
\end{array}\right)+\sum_{j=0}^{m-1} k_{j}(t) J L^{m-1-j}\left(\begin{array}{l}
-q \partial^{-1}\left(q^{2}+r^{2}\right)-r \\
-r \partial^{-1}\left(q^{2}+r^{2}\right)+q
\end{array}\right) \\
& +2 \sum_{j=0}^{m} k_{j}(t) J L^{m-j}\left(\begin{array}{l}
x q \\
x r
\end{array}\right)-k_{m}(t)\left(\begin{array}{l}
q \\
r
\end{array}\right) \\
= & J P_{n-1}(L)\left(\begin{array}{l}
q \\
r
\end{array}\right)+J Q_{m-1}(L)\left(\begin{array}{l}
-q \partial^{-1}\left(q^{2}+r^{2}\right)-r \\
-r \partial^{-1}\left(q^{2}+r^{2}\right)+q
\end{array}\right)+2 J \bar{Q}_{m}(L)\left(\begin{array}{l}
x q \\
x r
\end{array}\right) \\
& -k_{m}(t)\left(\begin{array}{l}
q \\
r
\end{array}\right),
\end{aligned}
$$


where

$$
\begin{aligned}
& P_{n-1}(L)=\sum_{j=0}^{n-1} \beta_{j}(t) L^{n-1-j}, \\
& Q_{m-1}(L)=\sum_{j=0}^{m-1} k_{j}(t) L^{m-1-j}, \\
& \bar{Q}_{m}(L)=2 \sum_{j=0}^{m} k_{j}(t) L^{m-j} .
\end{aligned}
$$

Taking

$$
\phi=J L J^{-1}=\left(\begin{array}{cc}
\partial q \partial^{-1} q & \partial+\partial q \partial^{-1} r \\
-\partial+\partial r \partial^{-1} q & \partial r \partial^{-1} r
\end{array}\right),
$$

then $J L^{n-1-j}=\phi^{n-1-j} J$. Hence, Eq. (24) again can be written as

$$
\begin{aligned}
U_{t_{n, m}=}= & \left(\begin{array}{l}
q \\
r
\end{array}\right)_{t_{n, m}} \\
= & P_{n-1}(\phi)\left(\begin{array}{l}
q_{x} \\
r_{x}
\end{array}\right)+Q_{m-1}(\phi)\left(\begin{array}{l}
-\left(q \partial^{-1}\left(q^{2}+r^{2}\right)\right)_{x}-r_{x} \\
-\left(r \partial^{-1}\left(q^{2}+r^{2}\right)\right)_{x}+q_{x}
\end{array}\right)+\bar{Q}_{m}(\phi) J\left(\begin{array}{c}
x q \\
x r
\end{array}\right) \\
& -k_{m}(t)\left(\begin{array}{l}
q \\
r
\end{array}\right) .
\end{aligned}
$$

\section{Expanding integrable models of the isospectral-nonisospectral integrable hierarchy}

For the Lie algebra $A_{1}$ presented previously, there are several enlarging Lie algebras in terms of [24]:

$$
\begin{aligned}
& A_{31}=\operatorname{span}\left\{g_{1}, g_{2}, g_{3}, g_{4}, g_{5}\right\}, \\
& g_{1}=\frac{1}{2}\left(\begin{array}{ccc}
1 & 0 & 0 \\
0 & -1 & 0 \\
0 & 0 & 0
\end{array}\right), \quad g_{2}=\frac{1}{2}\left(\begin{array}{lll}
0 & 1 & 0 \\
1 & 0 & 0 \\
0 & 0 & 0
\end{array}\right), \quad g_{3}=\frac{1}{2}\left(\begin{array}{ccc}
0 & 1 & 0 \\
-1 & 0 & 0 \\
0 & 0 & 0
\end{array}\right), \\
& g_{4}=\left(\begin{array}{lll}
0 & 0 & 1 \\
0 & 0 & 0 \\
0 & 0 & 0
\end{array}\right), \quad g_{5}=\left(\begin{array}{lll}
0 & 0 & 0 \\
0 & 0 & 1 \\
0 & 0 & 0
\end{array}\right), \\
& {\left[g_{1}, g_{2}\right]=g_{3}, \quad\left[g_{1}, g_{3}\right]=g_{2}, \quad\left[g_{2}, g_{3}\right]=-g_{1}, \quad\left[g_{1}, g_{4}\right]=\frac{1}{2} g_{5}, \quad\left[g_{4}, g_{5}\right]=0,} \\
& {\left[g_{1}, g_{5}\right]=\frac{1}{2} g_{4}, \quad\left[g_{2}, g_{4}\right]=-\left[g_{3}, g_{4}\right]=\frac{1}{2} g_{5}, \quad\left[g_{2}, g_{5}\right]=\frac{1}{2} g_{4}, \quad\left[g_{3}, g_{5}\right]=\frac{1}{2} g_{4},} \\
& A_{32}=\operatorname{span}\left\{f_{1}, f_{2}, f_{3}, f_{4}, f_{5}, f_{6}\right\}, \quad f_{3}=\left(\begin{array}{ll}
e & 0 \\
0 & e
\end{array}\right), \\
& f_{1}=\left(\begin{array}{ll}
h & 0 \\
0 & h
\end{array}\right), \quad f_{2}=\left(\begin{array}{ll}
f & 0 \\
0 & f
\end{array}\right), \quad
\end{aligned}
$$




$$
\begin{aligned}
& f_{4}=\left(\begin{array}{ll}
0 & h \\
0 & 0
\end{array}\right), \quad f_{5}=\left(\begin{array}{ll}
0 & e \\
0 & 0
\end{array}\right), \quad f_{6}=\left(\begin{array}{ll}
0 & f \\
0 & 0
\end{array}\right), \\
& {\left[f_{1}, f_{2}\right]=f_{3}, \quad\left[f_{1}, f_{3}\right]=f_{2}, \quad\left[f_{2}, f_{3}\right]=f_{1}, \quad\left[f_{1}, f_{4}\right]=0, \quad\left[f_{1}, f_{5}\right]=f_{6},} \\
& {\left[f_{1}, f_{6}\right]=f_{5}, \quad\left[f_{2}, f_{4}\right]=-f_{5}, \quad\left[f_{2}, f_{5}\right]=f_{4}, \quad\left[f_{2}, f_{6}\right]=0, \quad\left[f_{3}, f_{4}\right]=-f_{6},} \\
& {\left[f_{3}, f_{5}\right]=0, \quad\left[f_{3}, f_{6}\right]=-f_{4}, \quad\left[f_{4}, f_{5}\right]=\left[f_{4}, f_{6}\right]=\left[f_{5}, f_{6}\right]=0 .}
\end{aligned}
$$

Denoting $\tilde{g}_{1}=\operatorname{span}\left\{g_{1}, g_{2}, g_{3}\right\}, \tilde{g}_{2}=\operatorname{span}\left\{g_{4}, g_{5}\right\}$, it is easy to see that

$$
A_{31}=\tilde{g}_{1} \oplus \tilde{g}_{2}, \quad \tilde{g}_{1} \cong A_{1}, \quad\left[\tilde{g}_{1}, \tilde{g}_{2}\right] \subset \tilde{g}_{2},
$$

where $A_{1}=\operatorname{span}\{h, e, f\}, h=\frac{1}{2}\left(\begin{array}{cc}1 & 0 \\ 0 & -1\end{array}\right), e=\frac{1}{2}\left(\begin{array}{ll}0 & 1 \\ 1 & 0\end{array}\right), f=\frac{1}{2}\left(\begin{array}{cc}0 & 1 \\ -1 & 0\end{array}\right)$.

Similarly, noting $\tilde{f}_{1}=\operatorname{span}\left\{f_{1}, f_{2}, f_{3}\right\}, \tilde{f}_{2}=\operatorname{span}\left\{f_{4}, f_{5}, f_{6}\right\}$, then we find

$$
A_{31}=\tilde{f}_{1} \oplus \tilde{f}_{2}, \quad \tilde{f}_{1} \cong A_{1}, \quad\left[\tilde{f}_{1}, \tilde{f}_{2}\right] \subset \tilde{f}_{2} .
$$

The Lie subalgebras $\tilde{g}_{2}, \tilde{f}_{2}$, are all ideals of $A_{31}$ and $A_{31}$, respectively. Although the Lie subalgebras $\tilde{g}_{1}, \tilde{f}_{1}$ are all isomorphic to the Lie algebra $A_{1}$, the integrable systems obtained by using $\tilde{g}_{1}$ are different from those given by the use of $\tilde{f}_{1}$. The difference between them may be positive and negative. In the following, we can only apply the Lie algebra $A_{32}$ to investigate expanding integrable hierarchy of Eq. (11), while $A_{31}$ fails because there is not a resulting loop algebra corresponding to the loop algebra $\tilde{A}_{1}$. The loop algebra $\tilde{A}_{32}$ corresponding to the Lie algebra $A_{32}$ reads

$$
\tilde{A}_{32}=\operatorname{span}\left\{f_{1}(n), \ldots, f_{6}(n)\right\},
$$

where

$$
\begin{array}{ll}
f_{1}(n)=f_{1} \lambda^{2 n+1}, & f_{2}(n)=f_{2} \lambda^{2 n}, \quad f_{3}(n)=f_{3} \lambda^{2 n+1}, \\
f_{4}(n)=f_{4} \lambda^{2 n+1}, & f_{5}(n)=f_{5} \lambda^{2 n+1}, \quad f_{6}(n)=f_{6} \lambda^{2 n}, \quad n \in Z,
\end{array}
$$

along with the commutative relations

$$
\begin{array}{ll}
{\left[f_{1}(m), f_{2}(n)\right]=f_{3}(m+n),} & {\left[f_{1}(m), f_{3}(n)\right]=f_{2}(m+n+1),} \\
{\left[f_{2}(m), f_{3}(n)\right]=f_{1}(m+n),} & {\left[f_{1}(m), f_{4}(n)\right]=0, \quad\left[f_{1}(m), f_{5}(n)\right]=f_{6}(m+n+1),} \\
{\left[f_{1}(m), f_{6}(n)\right]=f_{5}(m+n),} & {\left[f_{2}(m), f_{4}(n)\right]=-f_{5}(m+n),} \\
{\left[f_{2}(m), f_{5}(n)\right]=f_{4}(m+n),} & {\left[f_{2}(m), f_{6}(n)\right]=0, \quad\left[f_{3}(m), f_{4}(n)\right]=-f_{6}(m+n+1),} \\
{\left[f_{3}(m), f_{4}(n)\right]=0, \quad\left[f_{3}(m), f_{6}(n)\right]=f_{4}(m+n),} & \\
{\left[f_{4}(m), f_{5}(n)\right]=\left[f_{4}(m), f_{6}(n)\right]=\left[f_{5}(m), f_{6}(n)\right]=0 .}
\end{array}
$$

\subsection{An isospectral expanding integrable model}

Applying the loop algebra $\tilde{A}_{32}$, we introduce the two linear spectral problems

$$
\left\{\begin{array}{l}
\varphi_{t}=\bar{U} \varphi, \quad \lambda_{t}=0 \\
\bar{U}=f_{2}(1)+q f_{3}(0)+r f_{1}(0)+u_{1} f_{4}(0)+u_{2} f_{5}(0)
\end{array}\right.
$$




$$
\left\{\begin{array}{l}
\varphi_{t}=\bar{V} \varphi=\left(\bar{V}_{1}+\bar{V}_{2}\right) \varphi \\
\bar{V}_{1}=\sum_{i=0}^{\infty}\left(a_{i} f_{1}(-i)+c_{i} f_{2}(-i)+b_{i} f_{3}(-i)+d_{i} f_{4}(-i)+e_{i} f_{5}(-i)+h_{i} f_{6}(-i)\right), \\
\bar{V}_{2}=\sum_{j=0}^{\infty}\left(\bar{a}_{j} f_{1}(-j)+\bar{c}_{j} f_{2}(-j)+\bar{b}_{j} f_{3}(-j)+\bar{d}_{j} f_{4}(-j)+\bar{e}_{j} f_{5}(-j)+\bar{h}_{j} f_{6}(-j)\right) .
\end{array}\right.
$$

First of all, we consider isospectral expanding integrable hierarchy corresponding to the Guo hierarchy. In terms of the Tu scheme, we solve the following matrix equation for $V_{1}$ :

$$
\bar{V}_{1, x}=\left[\bar{U}, \bar{V}_{1}\right]
$$

which admits the recurrence relations

$$
\begin{aligned}
& \left\{\begin{array}{l}
a_{i x}=b_{i+1}-q c_{i}, \\
c_{i x}=-q a_{i+1}+r b_{i+1}, \\
b_{i x}=-a_{i+1}+r c_{i}, \\
d_{i x}=e_{i+1}-q h_{i}-u_{2} c_{i}, \\
e_{i x}=-d_{i+1}+r h_{i}+u_{1} c_{i}, \\
h_{i x}=-q d_{i+1}+r e_{i+1}+u_{1} b_{i+1}-u_{2} a_{i+1}
\end{array}\right. \\
& \Rightarrow\left\{\begin{array}{l}
a_{i+1}=-b_{i x}+r c_{i}, \\
c_{i}=\partial^{-1}\left(r a_{i x}+q b_{i x}\right)+\beta_{i}(t), \\
b_{i+1}=a_{i x}+q c_{i}, \\
d_{i+1}=-e_{i x}+r h_{i}+u_{1} c_{i}, \\
e_{i+1}=d_{i x}+q h_{i}+u_{2} c_{i}, \\
h_{i}=\partial^{-1}\left(q e_{i x}+r d_{i x}+u_{1} a_{i x}+u_{2} b_{i x}\right)+\gamma_{i}(t) .
\end{array}\right.
\end{aligned}
$$

We take initial values by

$$
a_{0}=b_{0}=0, \quad c_{0}=\beta_{0}(t) q, \quad e_{0}=d_{0}=0, \quad h_{0}=\gamma_{0}(t) \text {. }
$$

Then from Eq. (30) one infers that

$$
\begin{aligned}
& a_{1}=\beta_{0}(t) r, \quad b_{1}=\beta_{0}(t) q, \\
& c_{1}=\frac{1}{2} \beta_{0}(t)\left(q^{2}+r^{2}\right)+\beta_{1}(t) \\
& a_{2}=-\beta_{0}(t) q_{x}+\frac{1}{2} \beta_{0}(t) r\left(q^{2}+r^{2}\right)+\beta_{1}(t) r \\
& b_{2}=\beta_{0}(t) r_{x}+\frac{1}{2} \beta_{0}(t) q\left(q^{2}+r^{2}\right)+\beta_{1}(t) q, \\
& c_{2}=\beta_{0}(t)\left(q r_{x}-q_{x} r\right)+\frac{3}{8} \beta_{0}(t)\left(q^{2}+r^{2}\right)^{2}+\frac{1}{2} \beta_{1}(t)\left(q^{2}+r^{2}\right), \\
& e_{1}=\gamma_{0}(t) q+\beta_{0}(t) u_{2}, \quad d_{1}=\gamma_{0}(t) r+\beta_{0}(t) u_{1}, \\
& h_{1}=\frac{1}{2} \gamma_{0}(t)\left(q^{2}+r^{2}\right)+\beta_{0}(t) q u_{2}+\beta_{0}(t) r u_{1}+\gamma_{1}(t),
\end{aligned}
$$




$$
\begin{aligned}
e_{2}= & \gamma_{0}(t) r_{x}+\beta_{0}(t) u_{1 x}+\frac{1}{2} \gamma_{0}(t)\left(q^{3}+q r^{2}\right)+\beta_{0}(t)\left(q^{2} u_{2}+q r u_{1}\right)+\frac{1}{2} \beta_{0}(t) u_{2}\left(q^{2}+r^{2}\right) \\
& +\gamma_{1}(t) q+\beta_{1}(t) u_{2}, \\
d_{2}= & -\gamma_{0}(t) q_{x}-\beta_{0}(t) u_{2 x}+\frac{1}{2} \gamma_{0}(t)\left(q^{2} r+r^{3}\right)+\beta_{0}(t)\left(r^{2} u_{1}+q r u_{2}\right)+\frac{1}{2} \beta_{0}(t) u_{1}\left(q^{2}+r^{2}\right) \\
& +\gamma_{1}(t) r+\beta_{1}(t) u_{1},
\end{aligned}
$$

\section{Denoting}

$$
\bar{V}_{1,+}^{n}=\sum_{i=0}^{n}\left(a_{i} f_{1}(-i)+c_{i} f_{2}(-i)+b_{i} f_{3}(-i)+d_{i} f_{4}(-i)+e_{i} f_{5}(-i)+h_{i} f_{6}(-i)\right) \lambda^{2 n}=\lambda^{2 n} \bar{V}-\bar{V}_{1,-}^{n},
$$

then Eq. (29) can be written as

$$
-\left(\bar{V}_{1,+}^{(n)}\right)_{x}+\left[\bar{U}, V_{1,+}^{(n)}\right]=\left(\bar{V}_{1,-}^{(n)}\right)_{x}-\left[\bar{U}, V_{1,-}^{(n)}\right] .
$$

The gradations of the left-hand side are $\geq 0$, those of the right-hand side are $\leq 1$. Therefore, we get

$$
\begin{aligned}
-\left(V_{1,+}^{(n)}\right)_{x}+\left[U, V_{1,+}^{(n)}\right]= & a_{n+1} f_{3}(0)-b_{n+1} f_{1}(0)+\left(q a_{n+1}-r b_{n+1}\right) f_{2}(0)+d_{n+1} f_{5}(0) \\
& -e_{n+1} f_{4}(0)+\left(q d_{n+1}-r e_{n+1}-u_{1} b_{n+1}+u_{2} a_{n+1}\right) f_{6}(0) .
\end{aligned}
$$

Assume that $V_{1}^{(n)}=V_{1,+}^{(n)}-c_{n} f_{2}(0)-h_{n} f_{6}(0)$, one infers again

$$
\begin{aligned}
-\left(\bar{V}_{1}^{(n)}\right)_{x}+\left[\bar{U}, \bar{V}_{1}^{(n)}\right]= & -\left(\bar{V}_{1,+}^{(n)}\right)_{x}+\left[\bar{U}, \bar{V}_{1,+}^{(n)}\right]+c_{n x} f_{2}(0)+\left[\bar{U},-c_{n} f_{2}(0)\right]+h_{n x} f_{6}(0) \\
& +\left[\bar{U},-h_{n} f_{6}(0)\right] \\
= & a_{n+1} f_{3}(0)-b_{n+1} f_{1}(0)+d_{n+1} f_{5}(0)-e_{n+1} f_{4}(0)+q c_{n} f_{1}(0) \\
& +q h_{n} f_{4}(0)-r c_{n} f_{3}(0)-r h_{n} f_{5}(0)-u_{1} c_{n} f_{5}(0)+u_{2} c_{n} f_{4}(0) \\
= & \left(-b_{n+1}+q c_{n}\right) f_{1}(0)+\left(a_{n+1}-r c_{n}\right) f_{3}(0)+\left(-e_{n+1}+u_{2} c_{n}+q h_{n}\right) f_{4}(0) \\
& +\left(d_{n+1}-u_{1} c_{n}-r h_{n}\right) f_{5}(0) \\
= & -a_{n x} f_{1}(0)-b_{n x} f_{3}(0)-d_{n x} f_{4}(0)-e_{n x} f_{5}(0)
\end{aligned}
$$

Hence, the zero curvature equation

$$
\bar{U}_{t}-\bar{V}_{x}^{(n)}+\left[\bar{U}, \bar{V}^{(n)}\right]=0,
$$

gives an isospectral integrable hierarchy

$$
\left(\begin{array}{c}
q \\
r \\
u_{1} \\
u_{2}
\end{array}\right)_{t_{n}}=\left(\begin{array}{l}
b_{n} \\
a_{n} \\
d_{n} \\
e_{n}
\end{array}\right)_{x}=\left(\begin{array}{llll}
\partial & 0 & 0 & 0 \\
0 & \partial & 0 & 0 \\
0 & 0 & \partial & 0 \\
0 & 0 & 0 & \partial
\end{array}\right)\left(\begin{array}{c}
b_{n} \\
a_{n} \\
d_{n} \\
e_{n}
\end{array}\right)=\bar{J}\left(\begin{array}{l}
b_{n} \\
a_{n} \\
d_{n} \\
e_{n}
\end{array}\right) .
$$


Taking $u_{1}=u_{2}=0$, Eq. (32) reduces to the Guo hierarchy Eq. (3). Thus, Eq. (32) is its expanding integrable hierarchy.

Case 1: when $n=1$, Eq. (32) reduces to

$$
\left\{\begin{array}{l}
q_{t}=\beta_{0}(t) q_{x} \\
r_{t}=\beta_{0}(t) r_{x} \\
u_{1 t}=\gamma_{0}(t) r_{x}+\beta_{0}(t) u_{1 x} \\
u_{2 t}=\gamma_{0}(t) q_{x}+\beta_{0}(t) u_{2 x}
\end{array}\right.
$$

Set $q=r=m, u_{1}=u_{2}=n$, then we get

$$
\left\{\begin{array}{l}
m_{t}=\beta_{0}(t) m_{x}, \\
n_{t}=\gamma_{0}(t) m_{x}+\beta_{0}(t) n_{x},
\end{array}\right.
$$

which is solvable.

Case 2: when $n=2$, Eq. (32) becomes

$$
\left\{\begin{aligned}
q_{t}= & \beta_{0}(t) r_{x x}+\frac{1}{2} \beta_{0}(t)\left(q\left(q^{2}+r^{2}\right)\right)_{x}+\beta_{0}(t) q_{x}, \\
r_{t}= & -\beta_{0}(t) q_{x x}+\frac{1}{2} \beta_{0}(t)\left(r\left(q^{2}+r^{2}\right)\right)_{x}+\beta_{1}(t) r_{x}, \\
u_{1 t}= & -\gamma_{0}(t) q_{x x}-\beta_{0}(t) u_{2 x x}+\frac{1}{2} \gamma_{0}(t)\left(q^{2} r+r^{3}\right)_{x}+\beta_{0}(t)\left(q r u_{2}+r^{2} u_{1}\right)_{x} \\
& +\frac{1}{2} \beta_{0}(t)\left(u_{1}\left(q^{2}+r^{2}\right)\right)_{x}+\gamma_{1}(t) r_{x}+\beta_{1}(t) u_{1 x}, \\
u_{2 t}= & \gamma_{0}(t) r_{x x}+\beta_{0}(t) u_{1 x x}+\frac{1}{2} \gamma_{0}(t)\left(q^{3}+q r^{2}\right)_{x}+\beta_{0}(t)\left(q^{2} u_{2}+r q u_{1}\right)_{x} \\
& +\frac{1}{2} \beta_{0}(t)\left(u_{2}\left(q^{2}+r^{2}\right)\right)_{x}+\gamma_{1}(t) q_{x}+\beta_{1}(t) u_{2 x} .
\end{aligned}\right.
$$

Let $\beta_{0}(t)=2, \beta_{1}(t)=0, \gamma_{0}(t)=2, \gamma_{1}(t)=0$. Equation (33) turns to

$$
\left\{\begin{array}{l}
q_{t}=2 r_{x x}+\left(q\left(q^{2}+r^{2}\right)\right)_{x} \\
r_{t}=-2 q_{x x}+\left(r\left(q^{2}+r^{2}\right)\right)_{x} \\
u_{1 t}=-2 q_{x x}-2 u_{2 x x}+\left(q^{2} r+r^{3}\right)_{x}+2\left(q r u_{2}+r^{2} u_{1}\right)_{x}+\left(u_{1}\left(q^{2}+r^{2}\right)\right)_{x} \\
u_{2 t}=2 r_{x x}+2 u_{1 x x}+\left(q^{3}+q r^{2}\right)_{x}+2\left(q^{2} u_{2}+q r u_{1}\right)_{x}+\left(u_{2}\left(q^{2}+r^{2}\right)\right)_{x}
\end{array}\right.
$$

which is obviously an expanding integrable model of Eq. (18). Specially, set $q=r=0$, Eq. (34) reduces to

$$
v_{t t}=-4 v_{x x x x},
$$

which is a special Boussinesq equation, here $v=u_{2}$. If $q=r=1$, Eq. (34) becomes a linear integrable system

$$
\left\{\begin{array}{l}
u_{1, t}=-2 u_{2, x x}+4 u_{1, x}+2 u_{2, x}, \\
u_{2, t}=2 u_{1, x x}+2 u_{1, x}+4 u_{2, x} .
\end{array}\right.
$$




\subsection{A nonisospectral integrable model}

Setting $\lambda_{t}=\sum_{j \geq 0} k_{j}(t) \lambda^{1-2 j}$, and solving the equation for $V_{2}$,

$$
\bar{V}_{2, x}=\left[\bar{U}, \bar{V}_{2}\right]+\frac{\partial \bar{U}}{\partial \lambda} \lambda_{t}
$$

we have

$$
\left\{\begin{array}{l}
\bar{a}_{j x}=\bar{b}_{j+1}-q \bar{c}_{j}+k_{j}(t) r, \\
\bar{c}_{j x}=-q \bar{a}_{j+1}+r \bar{b}_{j+1}+2 k_{j+1}(t), \\
\bar{b}_{j x}=-\bar{a}_{j+1}+r \bar{c}_{j}+k_{j}(t) q, \\
\bar{d}_{j x}=\bar{e}_{j+1}-q \bar{h}_{j}-u_{2} \bar{c}_{j}+k_{j}(t) u_{1}, \\
\bar{e}_{j x}=-\bar{d}_{j+1}+r \bar{h}_{j}+u_{1} \bar{c}_{j}+k_{j}(t) u_{2}, \\
\bar{h}_{j x}=-q \bar{d}_{j+1}+r \bar{e}_{j+1}+u_{1} \bar{b}_{j+1}-u_{2} \bar{a}_{j+1},
\end{array}\right.
$$

from which we get

$$
\begin{aligned}
& \bar{c}_{j}=\partial^{-1}\left(q \bar{b}_{j x}+r \bar{a}_{j x}\right)-k_{j}(t) \partial^{-1}\left(q^{2}+r^{2}\right)+2 k_{j+1}(t) x, \\
& \left.\bar{h}_{j}=\partial^{-1}\left(q \bar{e}_{j x}+r \bar{d}_{j x}\right)+u_{1} \bar{a}_{j x}+u_{2} \bar{b}_{j x}\right)-2 k_{j}(t) \partial^{-1}\left(r u_{1}+q u_{2}\right)+\alpha_{j}(t) .
\end{aligned}
$$

Taking $\bar{a}_{0}=\bar{b}_{0}=\bar{e}_{0}=\bar{d}_{0}=0$, one infers from Eq. (35) that

$$
\begin{aligned}
\bar{c}_{0}= & -k_{0}(t) \partial^{-1}\left(q^{2}+r^{2}\right)+2 k_{1}(t) x, \\
\bar{h}_{0}= & -2 k_{0}(t) \partial^{-1}\left(r u_{1}+q u_{2}\right)+\alpha_{0}(t), \\
\bar{a}_{1}= & -k_{0}(t) r \partial^{-1}\left(q^{2}+r^{2}\right)+2 k_{1}(t) x r+k_{0}(t) q, \\
\bar{b}_{1}= & -k_{0}(t) q \partial^{-1}\left(q^{2}+r^{2}\right)+2 k_{1}(t) x q-k_{0}(t) r \\
\bar{e}_{1}= & -2 k_{0}(t) q \partial^{-1}\left(r u_{1}+q u_{2}\right)+\alpha_{0}(t) q-k_{0}(t) u_{2} \partial^{-1}\left(q^{2}+r^{2}\right)+2 k_{1}(t) x u_{2}-k_{0}(t) u_{1}, \\
\bar{d}_{1}= & -2 k_{0}(t) r \partial^{-1}\left(r u_{1}+q u_{2}\right)+\alpha_{0}(t) r-k_{0}(t) u_{1} \partial^{-1}\left(q^{2}+r^{2}\right)+2 k_{1}(t) x u_{1}+k_{0}(t) u_{2}, \\
\bar{h}_{1}= & -k_{0}(t)\left(q^{2}+r^{2}\right) \partial^{-1}\left(r u_{1}+q u_{2}\right)-k_{0}(t)\left(r u_{1}+q u_{2}\right) \partial^{-1}\left(q^{2}+r^{2}\right)+2 k_{1}(t) x\left(r u_{1}+q u_{2}\right) \\
& +k_{0}(t) \partial^{-1}\left(u_{1} q_{x}-q u_{1 x}+r u_{2 x}-u_{2} r_{x}\right)+\frac{\alpha_{0}(t)}{2}\left(q^{2}+r^{2}\right)+\alpha_{1}(t),
\end{aligned}
$$

Assume

$$
\begin{aligned}
\bar{V}_{2,+}^{(m)}= & \sum_{j=0}^{m}\left(\bar{a}_{j} f_{1}(m-j)+\bar{c}_{j} f_{2}(m-j)+\bar{b}_{j} f_{3}(m-j)+\bar{d}_{j} f_{4}(m-j)+\bar{e}_{j} f_{5}(m-j)\right. \\
& \left.+\bar{h}_{j} f_{6}(m-j)\right) \\
= & \lambda^{2 m} \bar{V}_{2}-\bar{V}_{2,-}^{(m)} \\
\lambda_{t,+}^{(m)}= & \sum_{j=0}^{m} k_{j}(t) \lambda^{2 m-2 j+1}=\lambda^{2 m} \lambda_{t}-\lambda_{t,-}^{(m)}
\end{aligned}
$$


then we can compute that

$$
\begin{aligned}
- & \left(\bar{V}_{2,+}^{(m)}\right)_{x}+\left[\bar{U}, \bar{V}_{2,+}^{(m)}\right]+\frac{\partial \bar{U}}{\partial \lambda} \lambda_{t,+}^{(m)} \\
= & \left(\bar{V}_{2,-}^{(m)}\right)_{x}-\left[\bar{U}, \bar{V}_{2,-}^{(m)}\right]-\frac{\partial \bar{U}}{\partial \lambda} \lambda_{t,-}^{(m)} \\
= & \bar{a}_{m+1} f_{3}(0)-\bar{b}_{m+1} f_{1}(0)+\bar{d}_{m+1} f_{5}(0)-\bar{e}_{m+1} f_{4}(0)+q \bar{a}_{m+1} f_{2}(0)+q \bar{d}_{m+1} f_{6}(0) \\
& \quad-r \bar{b}_{m+1} f_{2}(0)-r \bar{e}_{m+1} f_{6}(0)-u_{1} \bar{b}_{m+1} f_{6}(0)+u_{2} \bar{a}_{m+1} f_{6}(0)-2 k_{m+1}(t) f_{2}(0)
\end{aligned}
$$

Denoting $\bar{V}_{2}^{(m)}=\bar{V}_{2,+}^{(m)}-\bar{c}_{m} f_{2}(0)-\bar{h}_{m} f_{6}(0)$, a direct calculation yields

$$
-\bar{V}_{2}^{(m)}+\left[\bar{U}, \bar{V}_{2}^{(m)}\right]+\frac{\partial \bar{U}}{\partial \lambda} \lambda_{t,+}^{(m)}=-\bar{a}_{m x} f_{1}(0)-\bar{b}_{m x} f_{3}(0)-\bar{d}_{m x} f_{4}(0)-\bar{e}_{m x} f_{5}(0)
$$

The zero curvature equation

$$
\frac{\partial \bar{U}}{\partial u} u_{t}+\frac{\partial \bar{U}}{\partial \lambda} \lambda_{t,+}^{(m)}-\bar{V}_{2, x}^{(m)}+\left[\bar{U}, \bar{V}_{2}^{(m)}\right]=0,
$$

leads to the isospectral integrable hierarchy

$$
\left(\begin{array}{c}
q \\
r \\
u_{1} \\
u_{2}
\end{array}\right)_{t_{m}}=\left(\begin{array}{l}
\bar{b}_{m x} \\
\bar{a}_{m x} \\
\bar{d}_{m x} \\
\bar{e}_{m x}
\end{array}\right)=\bar{J}\left(\begin{array}{l}
\bar{b}_{m} \\
\bar{a}_{m} \\
\bar{d}_{m} \\
\bar{e}_{m}
\end{array}\right)
$$

Taking $u_{1}=u_{2}=0$, Eq. (37) reduces to the isospectral integrable hierarchy Eq. (26) (the case $P_{n-1}(\phi)=0$ in Eq. (26).

When $n=1$, Eq. (37) turns to

$$
\left\{\begin{aligned}
q_{t}= & -k_{0}(t)\left(q \partial^{-1}\left(q^{2}+r^{2}\right)\right)_{x}+2 k_{1}(t)(x q)_{x}-k_{0}(t) r_{x} \\
r_{t}= & -k_{0}(t)\left(r \partial^{-1}\left(q^{2}+r^{2}\right)\right)_{x}+2 k_{1}(t)(x r)_{x}+k_{0}(t) q_{x} \\
u_{1 t}= & -2 k_{0}(t)\left(r \partial^{-1}\left(r u_{1}+q u_{2}\right)\right)_{x}+\alpha_{0}(t) r_{x}-k_{0}(t)\left(u_{1} \partial^{-1}\left(q^{2}+r^{2}\right)\right)_{x} \\
& +2 k_{1}(t)\left(x u_{1}\right)_{x}+k_{0}(t) u_{2 x} \\
u_{2 t}= & -2 k_{0}(t)\left(q \partial^{-1}\left(r u_{1}+q u_{2}\right)\right)_{x}+\alpha_{0}(t) q_{x}-k_{0}(t)\left(u_{2} \partial^{-1}\left(q^{2}+r^{2}\right)\right)_{x} \\
& +2 k_{1}(t)\left(x u_{2}\right)_{x}-k_{0}(t) u_{1 x}
\end{aligned}\right.
$$

Let $q^{2}+r^{2}=\rho_{x}, r u_{1}+q u_{2}=\sigma_{x}$, then Eq. (38) becomes a nonlocal expanding integrable model of Eq. (13):

$$
\left\{\begin{array}{l}
q_{t}=-k_{0}(t)(q \rho)_{x}+2 k_{1}(t)(x q)_{x}-k_{0}(t) r_{x} \\
r_{t}=-k_{0}(t)(r \rho)_{x}+2 k_{1}(t)(x r)_{x}+k_{0}(t) q_{x} \\
u_{1 t}=-2 k_{0}(t)(r \sigma)_{x}+\alpha_{0}(t) r_{x}-k_{0}(t)\left(u_{1} \rho\right)_{x}+2 k_{1}(t)\left(x u_{1}\right)_{x}+k_{0}(t) u_{2 x} \\
u_{2 t}=-2 k_{0}(t)(q \sigma)_{x}+\alpha_{0}(t) q_{x}-k_{0}(t)\left(u_{2} \sigma\right)_{x}+2 k_{1}(t)\left(x u_{2}\right)_{x}-k_{0}(t) u_{1 x}
\end{array}\right.
$$

For the Cauchy problem, blow-up phenomena could be investigated according to the method in [29], we shall consider the problem in another paper. 


\section{A Bäcklund transformation}

Tian and Zhang [30] once discussed the Bäcklund transformations of the AKNS hierarchy of evolution equations. The so-called Bäcklund transformation means a map from a solution of an equation to another solution of the same equation or another equation. In the isospectral case it is an auto-Bäcklund transformation, but in the nonisospectral case it is not an auto-Bäcklund transformation. It is easy to find the spectral matrix of the AKNS hierarchy to be

$$
U_{1}=\left(\begin{array}{cc}
-i \lambda & q \\
r & i \lambda
\end{array}\right)
$$

while that of the Guo hierarchy is

$$
U_{2}=\frac{1}{2}\left(\begin{array}{cc}
\lambda r & \lambda^{2}+\lambda q \\
-\lambda^{2}+\lambda q & -\lambda r
\end{array}\right) \text {. }
$$

$U_{2}$ is more complicated than $U_{1}$. Therefore, we could conclude the Bäcklund transformations of the isospectral and the nonisospectral Guo hierarchies have more tedious and complicated forms than those of the AKNS hierarchy. In the section, we only investigate the Bäcklund transformation of the isospectral Guo hierarchy. As for the nonisospectral case, we do not further discuss it again.

Denoting $\varphi_{1}=\left(\varphi_{11}, \varphi_{12}\right), \varphi_{2}=\left(\varphi_{21}, \varphi_{22}\right)$, Eq. (1) can be written as

$$
\left\{\begin{array}{l}
\varphi_{1 x}=\frac{1}{2} \lambda r \varphi_{1}+\frac{1}{2}\left(\lambda^{2}+\lambda q\right) \varphi_{2}, \\
\varphi_{2 x}=-\frac{1}{2} \lambda r \varphi_{2}+\frac{1}{2}\left(-\lambda^{2}+\lambda q\right) \varphi_{1} .
\end{array}\right.
$$

Equation (2) can be given again by

$$
\left(\begin{array}{c}
\varphi_{1 t} \\
\varphi_{2 t}
\end{array}\right)=\left(\begin{array}{cc}
A_{n} & B_{n} \\
C_{n} & -A_{n}
\end{array}\right)\left(\begin{array}{l}
\varphi_{1} \\
\varphi_{2}
\end{array}\right),
$$

where

$$
\begin{aligned}
& A_{n}=\frac{1}{2} \sum_{i=0}^{n} a_{i} \lambda^{2 n-2 i+1}, \\
& B_{n}=\frac{1}{2} \sum_{i=0}^{n}\left(b_{i} \lambda+c_{i}\right) \lambda^{2 n-2 i+1}-\frac{1}{2} c_{n}, \\
& C_{n}=\partial^{-1}\left(r A_{n x}+q B_{n x}\right) .
\end{aligned}
$$

We define an inner product in real-number space $R^{2}: \forall a=\left(a_{1}, a_{2}\right)^{T}, b=\left(b_{1}, b_{2}\right)^{T} \in R^{2}$, $\langle a, b\rangle=a^{T} b=\left(a_{1} b_{1}+a_{2} b_{2}\right)$. When investigating the conservation laws of some integrable hierarchy of evolution equations, we usually suppose that $\Gamma=\frac{\varphi_{2}}{\varphi_{1}}$ (see [22]). Here we want to seek a Bäcklund transformation of integrable hierarchies, so a transform between Lax pairs of linear spectral problem of integrable hierarchies and Lax pairs in Riccati forms is 
established as follows:

$$
\xi_{j}=\frac{v_{j} \varphi_{2}^{T}}{v_{j} \varphi_{1}^{T}}=: \frac{\left(\mu_{j}, v_{j}\right) \varphi_{2}^{T}}{\left(\mu_{j}, v_{j}\right) \varphi_{1}^{T}}=\frac{\mu_{j} \varphi_{21}\left(x, t, \lambda_{j}\right)+v_{j} \varphi_{22}\left(x, t, \lambda_{j}\right)}{\mu_{j} \varphi_{11}\left(x, t, \lambda_{j}\right)+v_{j} \varphi_{12}\left(x, t, \lambda_{j}\right)}, \quad j=1,2,
$$

where $\lambda_{i} \neq \lambda_{j}$, when $i \neq j,\left|\mu_{j}\right|+\left|v_{j}\right| \neq 0$.

Remark 2 Applying the idea we could get a transform like Eq. (41) when spectral matrices are of high orders, such as $3 \times 3$ matrices and $4 \times 4$ matrices.

Hence, we find

$$
\xi_{i x}=\frac{1}{2}\left(-\lambda^{2}+\lambda q\right)-\lambda r \xi_{j}-\frac{1}{2}\left(\lambda^{2}+\lambda q\right) \xi_{j}^{2} .
$$

Similarly, the linear spectral problem Eq. (40) can lead to

$$
\xi_{j t}=\frac{\varphi_{2 t}}{\varphi_{1}}-\frac{\varphi_{2}}{\varphi_{1}} \frac{\varphi_{1 t}}{\varphi_{1}}=C_{n}-2 A_{n} \xi_{j}-B_{n} \xi_{j}^{2}
$$

Note that $f=\frac{1}{\lambda_{1} \lambda_{2}\left(\xi_{2}^{2}-\xi_{1}^{2}\right)}, g=\frac{1}{\lambda_{1} \lambda_{2}\left(\xi_{2}-\xi_{1}\right)\left(1+\xi_{1} \xi_{2}\right)}$.

Define

$$
T=\left(\begin{array}{cc}
f\left[2 \lambda_{2} \partial+2 \lambda_{1} \lambda_{2}\left(r+\left(\lambda_{1}+q\right) \xi_{1}\right)\right] & -2 f\left[\lambda_{1} \partial+\lambda_{1} \lambda_{2}\left(r+\left(\lambda_{2}+q\right) \xi_{1}\right)\right] \\
g\left(1-\xi_{2}^{2}\right)\left[\lambda_{2} \partial+\lambda_{1} \lambda_{2}\left(r+\left(\lambda_{1}+q\right) \xi_{1}\right)\right] & -g\left(1-\xi_{1}^{2}\right)\left[\lambda_{1} \partial+\lambda_{1} \lambda_{2}\left(r+\left(\lambda_{2}+q\right) \xi_{2}\right)\right]
\end{array}\right),
$$

after tedious computation we have

$$
\left(\begin{array}{l}
q_{x} \\
r_{x}
\end{array}\right)=T\left(\begin{array}{l}
\xi_{1 x} \\
\xi_{2 x}
\end{array}\right)
$$

It is easy to see that

$$
T\left(\begin{array}{l}
\xi_{1 t} \\
\xi_{2 t}
\end{array}\right)=\left(\begin{array}{l}
A \\
B
\end{array}\right)
$$

where

$$
\begin{aligned}
A= & f\left[2 \lambda_{2} \xi_{1 x t}+2 \lambda_{1} \lambda_{2} r \xi_{1 t}+2 \lambda_{1} \lambda_{2}\left(\lambda_{1}+q\right) \xi_{1} \xi_{1 t}-2 \lambda_{1} \xi_{2 t x}-2 \lambda_{1} \lambda_{2} r \xi_{2 t}\right. \\
& \left.-2 \lambda_{1} \lambda_{2}\left(\lambda_{2}+q\right) \xi_{2} \xi_{2 t}\right], \\
B= & g\left(1-\xi_{2}^{2}\right)\left[\lambda_{2} \xi_{1 t x}+\lambda_{1} \lambda_{2} \xi_{1 t}+\lambda_{1} \lambda_{2}\left(\lambda_{1}+q\right) \xi_{1} \xi_{1 t}\right] \\
& -g\left(1-\xi_{1}^{2}\right)\left[\lambda_{1} \xi_{2 t x}+\lambda_{1} \lambda_{2} r \xi_{2 t}+\lambda_{1} \lambda_{2}\left(\lambda_{2}+q\right) \xi_{2} \xi_{2 t}\right] .
\end{aligned}
$$

Since

$$
\begin{aligned}
& \xi_{1 x t}=\frac{1}{2} \lambda_{1} q_{t}-\lambda_{1} r_{t} \xi_{1}-\lambda_{1} r \xi_{1 t}-\frac{1}{2} \lambda_{1} q_{t} \xi_{1}^{2}-\left(\lambda_{1}^{2}+\lambda_{1} q\right) \xi_{1} \xi_{1 t}, \\
& \xi_{2 x t}=\frac{1}{2} \lambda_{2} q_{t}-\lambda_{2} r_{t} \xi_{2}-\lambda_{2} r \xi_{2 t}-\frac{1}{2} \lambda_{2} q_{t} \xi_{2}^{2}-\left(\lambda_{2}^{2}+\lambda_{2} q\right) \xi_{2} \xi_{2 t},
\end{aligned}
$$


substituting them into $A$ and $B$, we have

$$
A=q_{t}+\frac{2}{\xi_{1}+\xi_{2}} r_{t}, \quad B=\frac{1-\xi_{2}^{2}}{1+\xi_{1} \xi_{2}}\left[r_{t}+\frac{1}{2}\left(\xi_{1}+\xi_{2}\right) q_{t}\right] .
$$

Therefore, Eq. (45) can be written as

$$
T\left(\begin{array}{l}
\xi_{1 t} \\
\xi_{2 t}
\end{array}\right)=\left(\begin{array}{cc}
1 & \frac{2}{\xi_{1}+\xi_{2}} \\
\frac{\left(\xi_{1}+\xi_{2}\right)\left(1-\xi_{2}^{2}\right)}{1+\xi_{1} \xi_{2}} & \frac{1-\xi_{2}^{2}}{1+\xi_{1} \xi_{2}}
\end{array}\right)\left(\begin{array}{c}
q_{t} \\
r_{t}
\end{array}\right)
$$

Obviously,

$$
\operatorname{det}\left(\begin{array}{cc}
1 & \frac{2}{\xi_{1}+\xi_{2}} \\
\frac{\left(\xi_{1}+\xi_{2}\right)\left(1-\xi_{2}^{2}\right)}{1+\xi_{1} \xi_{2}} & \frac{1-\xi_{2}}{1+\xi_{1} \xi_{2}}
\end{array}\right)=\frac{\xi_{2}^{2}-1}{1+\xi_{1} \xi_{2}} \neq 0 \quad\left(\xi_{2} \neq \pm 1\right)
$$

Hence, when $\xi_{2} \neq \pm 1$, we find that

$$
\left(\begin{array}{l}
q_{t} \\
r_{t}
\end{array}\right)=\left(\begin{array}{cc}
1 & \frac{2}{\xi_{1}+\xi_{2}} \\
\frac{\left(\xi_{1}+\xi_{2}\right)\left(1-\xi_{2}^{2}\right)}{1+\xi_{1} \xi_{2}} & \frac{1-\xi_{2}^{2}}{1+\xi_{1} \xi_{2}}
\end{array}\right)^{-1} T\left(\begin{array}{l}
\xi_{1 t} \\
\xi_{2 t}
\end{array}\right)=: \tilde{T}\left(\begin{array}{l}
\xi_{1 t} \\
\xi_{2 t}
\end{array}\right)
$$

where

$$
\tilde{T}=\left(\begin{array}{cc}
1 & \frac{2}{\xi_{1}+\xi_{2}} \\
\frac{\left(\xi_{1}+\xi_{2}\right)\left(1-\xi_{2}^{2}\right)}{1+\xi_{1} \xi_{2}} & \frac{1-\xi_{2}}{1+\xi_{1} \xi_{2}}
\end{array}\right)^{-1} T=\left(\begin{array}{cc}
1 & -\frac{2+2 \xi_{1} \xi_{2}}{\left(\xi_{1}+\xi_{2}\right)\left(\xi_{2}^{2}-1\right)} \\
-\xi_{1}-\xi_{2} & \frac{1+\xi_{1} \xi_{2}}{\xi_{2}^{2}-1}
\end{array}\right) T .
$$

From Eq. (40), one has

$$
\begin{aligned}
\left(\begin{array}{c}
B_{n} \\
A_{n}
\end{array}\right)= & \frac{1}{2} \sum_{i=0}^{n}\left(\begin{array}{c}
b_{i} \\
a_{i}
\end{array}\right) \lambda^{2 n-2 i+1}+\frac{1}{2} \sum_{i=0}^{n}\left(\begin{array}{cc}
\partial^{-1} q \partial & 0 \\
0 & \partial^{-1} r \partial
\end{array}\right)\left(\begin{array}{l}
b_{i} \\
a_{i}
\end{array}\right) \lambda^{2 n-2 i} \\
& -\frac{1}{2} \sum_{i=0}^{n}\left(\begin{array}{cc}
\partial^{-1} q \partial & 0 \\
0 & \partial^{-1} r \partial
\end{array}\right)\left(\begin{array}{c}
b_{n} \\
a_{n}
\end{array}\right) \\
= & \frac{1}{2} \lambda^{2 n} \sum_{i=0}^{n} \lambda^{1-2 i} L^{i-1} J^{-1}\left(\begin{array}{l}
q_{x} \\
r_{x}
\end{array}\right)+\frac{1}{2} \lambda^{2 n} \sum_{i=0}^{n} \lambda^{-2 i}\left(\begin{array}{cc}
\partial^{-1} q \partial & 0 \\
0 & \partial^{-1} r \partial
\end{array}\right) L^{i-1} J^{-1}\left(\begin{array}{l}
q_{x} \\
r_{x}
\end{array}\right) \\
& -\frac{1}{2}\left(\begin{array}{cc}
\partial^{-1} q \partial & 0 \\
0 & \partial^{-1} r \partial
\end{array}\right) L^{n-1} J^{-1}\left(\begin{array}{l}
q_{x} \\
r_{x}
\end{array}\right) .
\end{aligned}
$$

Denote

$$
\frac{1}{2} L^{i-1} J^{-1}=:\left(\begin{array}{cc}
\phi_{1} & \phi_{2} \\
\phi_{3} & \phi_{4}
\end{array}\right), \quad \frac{1}{2} L^{n-1} J^{-1}=\left(\begin{array}{ll}
\varphi_{1} & \varphi_{2} \\
\varphi_{3} & \varphi_{4}
\end{array}\right),
$$

then we get

$$
\left(\begin{array}{l}
B_{n} \\
A_{n}
\end{array}\right)=\sum_{i=0}^{n} \lambda^{2 n-2 i+1}\left(\begin{array}{l}
\phi_{1} q_{x}+\phi_{2} r_{x} \\
\phi_{3} q_{x}+\phi_{4} r_{x}
\end{array}\right)+\sum_{i=0}^{n} \lambda^{2 n-2 i}\left(\begin{array}{cc}
\partial^{-1} q \partial & 0 \\
0 & \partial^{-1} r \partial
\end{array}\right)\left(\begin{array}{l}
\phi_{1} q_{x}+\phi_{2} r_{x} \\
\phi_{3} q_{x}+\phi_{4} r_{x}
\end{array}\right)
$$




$$
-\frac{1}{2}\left(\begin{array}{cc}
\partial^{-1} q \partial & 0 \\
0 & \partial^{-1} r \partial
\end{array}\right)\left(\begin{array}{l}
\varphi_{1} q_{x}+\varphi_{2} r_{x} \\
\varphi_{3} q_{x}+\varphi_{4} r_{x}
\end{array}\right),
$$

from which one gets

$$
\begin{aligned}
& A_{n}=\sum_{i=0}^{n} \lambda^{2 n-2 i+1}\left(\phi_{3} q_{x}+\phi_{4} r_{x}\right)+\sum_{i=0}^{n} \lambda^{2 n-2 i} \partial^{-1} r \partial\left(\phi_{3} q_{x}+\phi_{4} r_{x}\right)-\frac{1}{2} \partial^{-1} r \partial\left(\varphi_{3} q_{x}+\varphi_{4} r_{x}\right), \\
& B_{n}=\sum_{i=0}^{n} \lambda^{2 n-2 i+1}\left(\phi_{1} q_{x}+\phi_{2} r_{x}\right)+\sum_{i=0}^{n} \lambda^{2 n-2 i} \partial^{-1} q \partial\left(\phi_{1} q_{x}+\phi_{2} r_{x}\right)-\frac{1}{2} \partial^{-1} q \partial\left(\varphi_{1} q_{x}+\varphi_{2} r_{x}\right) .
\end{aligned}
$$

Hence, we infer

$$
\begin{aligned}
& \left(\begin{array}{l}
C_{n}\left(\lambda_{1}\right)-2 A_{n}\left(\lambda_{1}\right) \xi_{1}-B_{n}\left(\lambda_{1}\right) \xi_{1}^{2} \\
C_{n}\left(\lambda_{2}\right)-2 A_{n}\left(\lambda_{2}\right) \xi_{2}-B_{n}\left(\lambda_{2}\right) \xi_{2}^{2}
\end{array}\right) \\
& =\left(\begin{array}{l}
\left(\partial^{-1} r \partial-2 \xi_{1}\right) A_{n}\left(\lambda_{1}\right)+\left(\partial^{-1} q \partial-\xi_{1}^{2}\right) B_{n}\left(\lambda_{1}\right) \\
\left(\partial^{-1} r \partial-2 \xi_{2}\right) A_{n}\left(\lambda_{2}\right)+\left(\partial^{-1} q \partial-\xi_{2}^{2}\right) B_{n}\left(\lambda_{2}\right)
\end{array}\right) \\
& =\sum_{i=0}^{n}\left(\begin{array}{ll}
\left(\partial^{-1} q \partial-\xi_{1}^{2}\right) \lambda_{1}^{2 n-2 i}\left(\lambda_{1}+\partial^{-1} q \partial\right) & \left(\partial^{-1} r \partial-2 \xi_{1}\right) \lambda_{1}^{2 n-2 i}\left(\lambda_{1}+\partial^{-1} r \partial\right) \\
\left(\partial^{-1} q \partial-\xi_{2}^{2}\right) \lambda_{2}^{2 n-2 i}\left(\lambda_{2}+\partial^{-1} q \partial\right) & \left(\partial^{-1} r \partial-2 \xi_{2}\right) \lambda_{2}^{2 n-2 i}\left(\lambda_{2}+\partial^{-1} r \partial\right)
\end{array}\right) \\
& \quad \times L^{i-1} J^{-1}\left(\begin{array}{l}
q_{x} \\
r_{x}
\end{array}\right) \\
& \quad-\frac{1}{2}\left(\begin{array}{ll}
\left(\partial^{-1} q \partial-\xi_{1}^{2}\right) \partial^{-1} q \partial & \left(\partial^{-1} r \partial-2 \xi_{1}\right) \partial^{-1} r \partial \\
\left(\partial^{-1} q \partial-\xi_{2}^{2}\right) \partial^{-1} q \partial & \left(\partial^{-1} r \partial-2 \xi_{2}\right) \partial^{-1} r \partial
\end{array}\right) L^{n-1} J^{-1}\left(\begin{array}{l}
q_{x} \\
r_{x}
\end{array}\right) .
\end{aligned}
$$

Thus, Eq. (47) can be written as

$$
\begin{aligned}
\left(\begin{array}{l}
q_{t} \\
r_{t}
\end{array}\right)= & \left(\begin{array}{cc}
1 & -\frac{2+2 \xi_{1} \xi_{2}}{\left(\xi_{1}+\xi_{2}\right)\left(\xi_{2}^{2}-1\right)} \\
-\xi_{1}-\xi_{2} & \frac{1+\xi_{1} \xi_{2}}{\xi_{2}^{2}-1}
\end{array}\right) T \\
& \times\left[\sum_{i=0}^{n}\left(\begin{array}{cc}
\left(\partial^{-1} q \partial-\xi_{1}^{2}\right) \lambda_{1}^{2 n-2 i}\left(\lambda_{1}+\partial^{-1} q \partial\right) & \left(\partial^{-1} r \partial-2 \xi_{1}\right) \lambda_{1}^{2 n-2 i}\left(\lambda_{1}+\partial^{-1} r \partial\right) \\
\left(\partial^{-1} q \partial-\xi_{2}^{2}\right) \lambda_{2}^{2 n-2 i}\left(\lambda_{2}+\partial^{-1} q \partial\right) & \left(\partial^{-1} r \partial-2 \xi_{2}\right) \lambda_{2}^{2 n-2 i}\left(\lambda_{2}+\partial^{-1} r \partial\right)
\end{array}\right)\right. \\
& \left.\times L^{i-1}\left(\begin{array}{l}
q \\
r
\end{array}\right)-\frac{1}{2}\left(\begin{array}{cc}
\left(\partial^{-1} q \partial-\xi_{1}^{2}\right) \partial^{-1} q \partial & \left(\partial^{-1} r \partial-2 \xi_{1}\right) \partial^{-1} r \partial \\
\left(\partial^{-1} q \partial-\xi_{2}^{2}\right) \partial^{-1} q \partial & \left(\partial^{-1} r \partial-2 \xi_{2}\right) \partial^{-1} r \partial
\end{array}\right) L^{n-1}\left(\begin{array}{l}
q \\
r
\end{array}\right)\right] .
\end{aligned}
$$

Equation (47) indicates that the Guo hierarchy can be expressed by the solutions of the Lax pairs in the Riccati forms.

Applying the Riccati equation (43), we define a pair of new variables $\bar{q}, \bar{r}$ which satisfy the following equations:

$$
\left\{\begin{array}{l}
\xi_{1 x}=\frac{1}{2}\left(-\lambda_{2}^{2}+\lambda_{2} \bar{q}-\lambda_{2} \bar{r} \xi_{1}-\frac{1}{2}\left(\lambda_{2}^{2}+\lambda_{2} \bar{q}\right) \xi_{1}^{2}\right. \\
\xi_{2 x}=\frac{1}{2}\left(-\lambda_{1}^{2}+\lambda_{1} \bar{q}-\lambda_{1} \bar{r} \xi_{2}-\frac{1}{2}\left(\lambda_{1}^{2}+\lambda_{1} \bar{q}\right) \xi_{2}^{2}\right.
\end{array}\right.
$$

from which we can solve $\bar{q}, \bar{r}$ given by

$$
\bar{q}=\frac{\lambda_{1}^{2}-\lambda_{2}^{2}+\lambda_{2}^{2} \xi_{2}^{2}-\lambda_{1}^{2} \xi_{1}^{2}}{\lambda_{2} \lambda_{1}\left(\xi_{2}^{2}-\xi_{1}^{2}\right)} q+\frac{2\left(\lambda_{2}^{2} \xi_{2}-\lambda_{1}^{2} \xi_{1}\right)}{\lambda_{2} \lambda_{1}\left(\xi_{2}^{2}-\xi_{1}^{2}\right)} r+\frac{\lambda_{2}^{3}-\lambda_{1}^{3}+\lambda_{2}^{3} \xi_{2}^{2}-\lambda_{1}^{3} \xi_{1}^{2}}{\lambda_{2} \lambda_{1}\left(\xi_{2}^{2}-\xi_{1}^{2}\right)},
$$




$$
\begin{aligned}
\bar{r}= & \frac{\left(1-\xi_{1}^{2}\right)\left(1-\xi_{2}^{2}\right)\left(\lambda_{1}^{2}-\lambda_{2}^{2}\right)}{2 \lambda_{2} \lambda_{1}\left(\xi_{2}-\xi_{1}\right)\left(1+\xi_{1} \xi_{2}\right)} q+\frac{2 \lambda_{2}^{2} \xi_{2}\left(1-\xi_{1}^{2}\right)-2 \lambda_{1}^{2} \xi_{1}\left(1-\xi_{2}^{2}\right)}{2 \lambda_{2} \lambda_{1}\left(\xi_{2}-\xi_{1}\right)\left(1+\xi_{1} \xi_{2}\right)} r \\
& +\frac{\lambda_{2}^{3}\left(1-\xi_{1}^{2}\right)\left(1+\xi_{2}^{2}\right)-\lambda_{1}^{3}\left(1+\xi_{1}^{2}\right)\left(1-\xi_{2}^{2}\right)}{2 \lambda_{2} \lambda_{1}\left(\xi_{2}-\xi_{1}\right)\left(1+\xi_{2} \xi_{1}\right)} .
\end{aligned}
$$

Similar to the steps in [30], we can verify that Eq. (49), Eq. (50) is a set of solutions of the Guo hierarchy, here we omit the detailed computations due to the rather tedious calculations.

\section{Discussion on the conserved densities of the integrable system of equations (18)}

In this section, we only take the obtained integrable system of equations (18) as an example to illustrate how to generate conserved densities. Actually, we have obtained many integrable equations by the reduction of the hierarchy Eq. (11). By applying the analogous method we could work out their resulting conserved densities via the approach in [31].

For the partial differential system

$$
u_{i, t}+F_{i}\left(u_{j}, u_{j}^{(1)}, \ldots, u_{j}^{(n)}\right)=0, \quad i=1, \ldots, N ; j=1, \ldots, N
$$

Step 1: Determine the weights of variables and parameters.

We define the weight of a variable as the number of partial derivatives with respect to $x$ the variable carries, and the rank of a term as the total weight in terms of partial derivatives with respect to $x$.

(i) Take the $i$ th equation in Eq. (51) and denote the number of terms in the equation by $K_{i}$.

(ii) Compute the rank $r_{i, k}$ of the $k$ th term in the $i$ th equation

$$
r_{i, k}=d(x)+d(t) w\left(\partial_{t}\right)+\sum_{j=1}^{N} g\left(u_{j}\right) w\left(u_{j}\right)+\sum_{j=1}^{p} g\left(p_{j}\right) w\left(p_{j}\right), \quad k=1, \ldots, K_{i}
$$

where $g$ returns the degree of nonlinearity of its argument, $d$ returns the number of partial derivatives with respect to its argument.

(iii) Assume uniformity in rank in the $i$ th equation, form the linear system

$$
A_{i}=\left\{r_{i, 1}=r_{i, 2}=\cdots=r_{i, k_{i}}\right\}
$$

(iv) Gather the equations $A_{i}$ to form the global linear system $\mathcal{A}=\bigcup_{i=1}^{N} A_{i}$.

Step 2: Construct the form of the density.

Let $v=\left\{v_{1}, v_{2}, \ldots, v_{a}\right\}$ be the sorted list of all the variables with positive weights, but excluding $\partial_{t}$.

Form all monomials of rank $R$ or less by taking combinations of the variables in $v$. Set $\mathcal{B}_{0}=\{(1 ; 0)\}$. For $1 \leq q \leq Q$, we begin to compute the pair $\left\{T_{q, s} ; W_{q, s}\right\}$ in terms of the formula

$$
T_{q, s}=T_{q-1, m} v_{q}^{s}, W_{q, s}=W_{q-1, m}+s w\left(v_{q}\right) .
$$


Denote $B_{q, m}=\bigcup_{s=0}^{b_{q, m}}\left\{\left(T_{q, s} ; W_{q, s}\right)\right\}$, where $b_{q, m}=\left[\left[\frac{R-W_{q-1, m}}{w\left(v_{q}\right)}\right]\right], \mathcal{B}_{q}=\bigcup_{m=0}^{M-1} B_{q, m}$, here $M$ is the number of pairs in $\mathcal{B}_{q-1}$. We apply $\frac{\partial^{l}}{\partial x^{l}}$ to the term $T_{Q, S}$, provided $l=R-W_{Q, S}$ is integer. Then remove the terms from $\mathcal{B}_{q}$ that can be written as a total derivative with respect to $x$, or as a derivative up to terms kept prior in the set. Call the resulting set $\mathcal{I}$. Finally, set $\rho=$ $\sum_{i=1}^{\mu} c_{i} I(i)$, where $I(i)$ denotes the $i$ th element in $\mathcal{I}$, and $c_{i}$ are constants to be determined.

Step 3: Determine the unknown coefficients in the density $\rho$.

Recall the conservation law:

$$
D_{t} \rho+D_{x} J=0
$$

$J$ is called the flux, and $\rho$ is the conserved density. Equation (52) indicates that $D_{t} \rho=$ $D_{x}(-J)$. Hence, we first compute $D_{t} \rho$ and replace all $\left(u_{i, t}\right)^{j}, i=0,1, \ldots, N, j=0,1,2, \ldots$, using the evolution equations (51). The resulting expression, denoted by $E$, must equal $D_{x}(-J)$ for some function $J$. Next, apply the Euler operator to $E$ to get a linear system for the coefficients $c_{i}$ denoted by $S$. Solving $S$ for the $c_{i}$ yields the corresponding conserved densities.

In what follows, we consider the formats of conserved densities of Eq. (18) based on the above approach. It is easy to see that

$$
\begin{aligned}
& r_{1,1}=w(q)+w\left(\partial_{t}\right), \quad r_{1,2}=2+w(r), \quad r_{1,3}=1+3 w(q), \quad r_{2,3}=1+3 w(r) ; \\
& r_{1,4}=1+w(q)+2 w(r), \quad r_{2,1}=w(r)+w\left(\partial_{t}\right), \quad r_{2,2}=2+w(q), \\
& r_{2,4}=1+w(r)+2 w(q) .
\end{aligned}
$$

Set $r_{1,1}=r_{1,2}=r_{1,3}=r_{1,4}, r_{2,1}=r_{2,2}=r_{2,3}=r_{2,4}$, we have $w(q)=w(r)=\frac{1}{2}, w\left(\partial_{t}\right)=2$.

Remark 3 In the paper [31], the conserved densities of partial differential equations along with the integer number weights are investigated. As for the case of the rational number weights, there are no examples to give a detailed analysis. For the integrable system of equations (18), the weights of $q$ and $r$ are all a rational number, $\frac{1}{2}$, hence we have to face the difficulty of how to find the conserved densities of Eq. (18). Multiplying the first equation in Eq. (25) by $r$, and multiplying the second equation by $q$, then adding them together, we find that

$$
\left(q^{2}+r^{2}\right)_{t}=\left(4\left(q r_{x}-q_{x} r\right)+3\left(q^{2}+r^{2}\right)^{2}\right)_{x}
$$

which indicates that $q^{2}+r^{2}$ is a conserved density. In terms of this fact we want to search for the conserved density of Eq. (18) with weight being 1 . Take $v=\left\{u=q^{2}, v_{2}=r^{2}, v_{3}=q r\right\}$, $\mathcal{B}_{0}=\{(1 ; 0)\}$. Let us consider the case where $\operatorname{rank} R=4$.

For $q=1, m=0$ (here $q$ is not that in Eq. (18): $b_{1,0}=\left[\left[\frac{4-0}{1}\right]\right]=4, T_{1, s}=v_{1}^{s}, W_{1, s}=s w\left(v_{1}\right)=s$, where $s=0,1,2,3,4$. We obtain $\mathcal{B}_{1}=B_{1,0}=\left\{(1 ; 0),\left(q^{2} ; 1\right),\left(q^{4} ; 2\right),\left(q^{6} ; 3\right),\left(q^{8} ; 4\right)\right\}$.

For $q=2, m=0: b_{2,0}=\left[\left[\frac{4-W_{1,4}}{1}\right]\right]=4, T_{2, s}=r^{2 s}, W_{2, s}=W_{1,0}+s w\left(v_{2}\right)=s$, here $s=$ $0,1,2,3,4$. we have $B_{2,0}=\left\{(1 ; 0),\left(r^{2} ; 1\right),\left(r^{4} ; 2\right),\left(r^{6} ; 3\right),\left(r^{8} ; 4\right)\right\}$.

For $q=2, m=1: b_{2,1}=\left[\left[\frac{4-W_{1,1}}{1}\right]\right]=3, T_{2, s}=T_{1,1} v_{2}^{s}=q^{2} r^{2 s}, W_{2, s}=W_{1,1}+s=1+s$, where $s=0,1,2,3$. Thus, we get $B_{2,1}=\left\{\left(q^{2} ; 1\right),\left(q^{2} r^{2} ; 2\right),\left(q^{2} r^{4} ; 3\right),\left(q^{2} r^{6} ; 4\right)\right\}$.

For $q=2, m=2: b_{2,2}=\left[\left[\frac{4-W_{1,2}}{1}\right]\right]=2, T_{2, s}=T_{1,2} v_{2}^{s}=q^{4} r^{2 s}, W_{2, s}=W_{1,2}+s=2+s$, here $s=0,1,2$. We obtain $B_{2,2}=\left\{\left(q^{4} ; 2\right),\left(q^{4} r^{2} ; 3\right),\left(q^{4} r^{4} ; 4\right)\right\}$. 
For $q=2, m=3: b_{2,3}=\left[\left[\frac{4-3}{1}\right]\right]=1$, where $s=0,1, T_{2, s}=q^{6} r^{2 s}, W_{2, s}=W_{1,3}+s=3+s$, we have $B_{2,3}=\left\{\left(q^{6} ; 3\right),\left(q^{6} r^{2} ; 4\right)\right\}$.

For $q=2, m=4: b_{2,4}=0, T_{2, s}=T_{1,4} v_{2}^{s}=q^{8} v_{2}^{s}, W_{2, s}=4+s$, here $s=0$. Thus, one has $B_{2,4}=\left\{\left(q^{8} ; 4\right)\right\}$.

Hence, $\mathcal{B}_{2}=\left\{(1 ; 0),\left(q^{2} ; 1\right),\left(q^{4} ; 2\right),\left(q^{6} ; 3\right),\left(q^{8} ; 4\right),\left(r^{2} ; 1\right),\left(r^{4} ; 2\right),\left(r^{6} ; 3\right),\left(r^{8} ; 4\right),\left(q^{6} r^{2} ; 4\right),\left(q^{2} r^{2}\right.\right.$; $\left.2),\left(q^{2} r^{4} ; 3\right),\left(q^{2} r^{6} ; 4\right),\left(q^{4} r^{2} ; 3\right),\left(q^{4} r^{4} ; 4\right)\right\}$.

For $q=3, m=0: b_{3,0}=\left[\left[\frac{4-W_{2,0}}{w\left(v_{3}\right)}\right]\right]=4, T_{3, s}=T_{2,0} v_{3}^{s}=(q r)^{s}, W_{3, s}=W_{2,0}+s w\left(v_{3}\right)=s$, where $s=0,1,2,3,4$. So $B_{3,0}=\left\{(1 ; 0),(q r ; 1),\left(q^{2} r^{2} ; 2\right),\left(q^{3} r^{3} ; 3\right),\left(q^{4} r^{4} ; 4\right)\right\}$.

For $q=3, m=1: b_{3,1}=\left[\left[\frac{4-W_{2,1}}{1}\right]\right]=2, T_{3, s}=T_{2,1} v_{3}^{s}=q^{2} r^{2}(q r)^{s}, W_{3, s}=W_{2,1}+s=2+s$, where $s=0,1,2$. Hence, $B_{3,1}=\left\{\left(q^{2} r^{2} ; 2\right),\left(q^{3} r^{3} ; 3\right),\left(q^{4} r^{4} ; 4\right)\right\}$.

For $q=3, m=2: b_{3,2}=\left[\left[\frac{4-W_{2,2}}{1}\right]\right]=2, T_{3, s}=T_{2,2} v_{3}^{s}=q^{4} r^{4}(q r)^{s}, W_{3, s}=W_{2,2}+s=4+s$, $s=0,1,2$. Thus, we get $B_{3,2}=\left\{\left(q^{4} r^{4} ; 4\right)\right\}$.

As for $s=1,2$ and $q=3, m=3, \ldots, 14$, we would obtain the same pairs as the previous cases. Therefore, we have

$$
\begin{aligned}
\mathcal{B}_{3}= & \mathcal{G}=\mathcal{B}_{0} \cup \mathcal{B}_{2} \cup\left\{B_{3,0} \cup B_{3,1} \cup B_{2,2}\right\} \\
= & \left\{(1 ; 0),\left(q^{2} ; 1\right),\left(q^{4} ; 2\right),\left(q^{6} ; 3\right),\left(q^{8} ; 4\right),\left(r^{2} ; 1\right),\left(r^{4} ; 2\right),\left(r^{6} ; 3\right),\left(r^{8} ; 4\right),\left(q^{6} r^{2} ; 4\right),\right. \\
& \left.\left(q^{2} r^{2} ; 2\right),\left(q^{2} r^{4} ; 3\right),\left(q^{2} r^{6} ; 4\right),\left(q^{4} r^{2} ; 3\right),\left(q^{4} r^{4} ; 4\right),\left(q^{3} r^{3} ; 3\right)\right\} .
\end{aligned}
$$

Next, we apply derivatives to the first components of the pairs in $\mathcal{G}$. Computation of $l$ for each pair of $\mathcal{G}$ leaves us with

$$
l=4,3,2,1,0,3,2,1,0,0,2,1,0,1,0,2,1,0 .
$$

Gathering the terms that come from applying the indicated number $l$ of partial derivatives with respect to $x$ gives

$$
\begin{aligned}
\mathcal{H}= & \left\{0, q_{x} q_{x x}, q q_{x x x}, q^{2} q_{x}^{2}, q^{3} q_{x x}, q^{5} q_{x}, q^{8}, r_{x} r_{x x}, r r_{x x x}, r^{2} r_{x}, r^{3} r_{x x}, r^{5} r_{x}, r^{8}, q^{6} r^{2},\right. \\
& \left.q_{x}^{2} r^{2}, q q_{x x} r^{2}, q q_{x} r r_{x}, q^{2} r_{x}^{2}, q^{2} r r_{x x}, q q_{x} r^{4}, q^{2} r^{3} r_{x}, q^{2} r^{6}, q^{3} q_{x} r^{2}, q^{4} r r^{x}, q^{4} r^{4}\right\} .
\end{aligned}
$$

Removing from $\mathcal{H}$ the constant terms, the terms that can be written as a $x$-derivative or as a $x$-derivative up to terms retained earlier in the set $\mathcal{I}$, yields

$$
\begin{aligned}
\mathcal{I}= & \left\{q q_{x x x}, q^{3} q_{x x}, q^{2} q_{x}^{2}, q^{8}, r r_{x x x}, r^{2} r_{x}^{2}, r^{3} r_{x x}, r^{8}, q^{6} r^{2}, q_{x}^{2} r^{2}, q q_{x} r r_{x}, q^{2} r_{x}^{2},\right. \\
& \left.q^{2} r^{3} r_{x}, q^{2} r^{6}, q^{3} q_{x} r^{2}, q^{4} r^{4}\right\} .
\end{aligned}
$$

Let the form of the density with rank 4 be the following:

$$
\begin{aligned}
\rho= & c_{1} q q_{x x x}+c_{2} q^{3} q_{x x}+c_{3} q^{8}+c_{4} r r_{x x x}+c_{5} r^{3} r_{x x}+c_{6} r^{8}+c_{7} q^{6} r^{2}+c_{8} q_{x}^{2} r^{2}+c_{9} q q_{x} r r_{x} \\
& +c_{10} q^{2} r_{x}^{2}+c_{11} q^{2} r^{3} r_{x}+c_{12} q^{2} r^{6}+c_{13} q^{3} q_{x} r^{2}+c_{14} q^{4} r^{4}+c_{15} q^{2} q_{x}^{2}+c_{16} r^{2} r_{x}^{2}
\end{aligned}
$$

where $c_{i}=(i=1, \ldots, 16)$ are constants to be determined. Computation of $D_{t} \rho$ and replac$\operatorname{ing} q_{t}^{j}, r_{t}^{j}(j=0,1,2,3)$ by using Eq. (18) yield the function

$$
E=\left(2 c_{2} q^{3}+2 c_{1} q^{2} r+c_{4} q^{2} r+3 c_{4} r^{3}\right) r_{x x x x}+\left(3 c_{1} q^{3}+c_{1} q r^{2}+2 c_{4} q r^{2}-2 c_{5} r^{3}\right) q_{x x x x}
$$




$$
\begin{aligned}
& +\left(2 c_{2} q^{4} r+2 c_{13} q^{3} r^{2}+c_{5} q^{2} r^{3}+3 c_{5} r^{5}+4 c_{15} q^{2} q_{x}+8 c_{1} q^{2} r_{x}+c_{4} q^{2} r_{x}+8 c_{1} q q_{x} r\right. \\
& \left.+10 c_{4} q q_{x} r+2 c_{9} q r r_{x}+4 c_{8} q_{x} r^{2}+27 c_{4} r^{2} r_{x}-2 c_{4} q_{x x}\right) r_{x x x}+\left(3 c_{2} q^{5}+c_{2} q^{3} r^{2}\right. \\
& -2 c_{11} q^{2} r^{3}+2 c_{5} q r^{4}+27 c_{1} q^{2} q_{x}-4 c_{10} q^{2} r_{x}-2 c_{9} q q_{x} r+10 c_{1} q r r_{x}+8 c_{4} q r r_{x}+c_{1} q_{x} r^{2} \\
& \left.+8 c_{4} q_{x} r^{2}-4 c_{16} r^{2} r_{x}+2 c_{1} r_{x x}\right) q_{x x x}+\left(6 c_{1} q^{2}+18 c_{4} r^{2}\right) r_{x x}^{2}+\left(16 c_{3} q^{7}+\left(6 c_{2} q^{2}\right.\right. \\
& \left.+12 c_{1} q r+12 c_{4} q r-6 c_{5} r^{2}\right) q_{x x}+4 c_{15} q^{3} q_{x}+6 c_{2} q^{3} q_{x} r+c_{9} q^{3} q_{x} r+6 c_{13} q^{2} q_{x} r^{2} \\
& +6 c_{10} q^{2} r^{2} r_{x}+2 c_{16} q^{2} r^{2} r_{x}+3 c_{5} q^{2} r^{2} r_{x}+2 c_{9} q^{2} r^{2} r_{x}+12 c_{5} q q_{x} r^{3}+4 c_{8} q q_{x} r^{3} \\
& +3 c_{9} q_{x} r^{3}+4 c_{11} q r^{3} r_{x}+24 c_{1} q q_{x} r_{x}+2 c_{9} q_{x} r r_{x}+12 c_{7} q^{5} r^{2}+c_{11} q^{4} r^{3}+2 c_{13} q^{4} r^{3} \\
& +8 c_{14} q^{3} r^{4}+3 c_{11} q^{2} r^{5}+4 c_{12} q r^{6}+2 c_{10} q^{4} r_{x}+6 c_{2} q^{4} r_{x}+6 c_{16} r^{4} r_{x}+27 c_{5} r^{4} r_{x} \\
& \left.+4 c_{15} q q_{x}^{2}+4 c_{10} q r_{x}^{2}+12 c_{4} r q_{x}^{2}+36 c_{4} r r_{x}^{2}\right) r_{x x}+\left(18 c_{1} q^{2}+6 c_{4} r^{2}\right) q_{x x}^{2}+\left(-4 c_{13} q^{3} q_{x} r\right. \\
& +4 c_{10} q^{3} r r_{x}+12 c_{2} q^{3} r r_{x}+3 c_{9} q^{3} r r_{x}+2 c_{15} q^{2} q_{x} r^{2}+3 c_{2} q^{2} q_{x} r^{2}+6 c_{8} q^{2} q_{x} r^{2} \\
& +2 c_{9} q^{2} q_{x} r^{2}-6 c_{11} q^{2} r^{2} r_{x}+4 c_{16} q r^{3} r_{x}+6 c_{5} q r^{3} r_{x}+c_{9} q r^{3} r_{x}-2 c_{9} q q_{x} r_{x}-16 c_{6} r^{7} \\
& -4 c_{7} q^{6} r+3 c_{13} q^{5} r^{2}-8 c_{14} q^{4} r^{3}+2 c_{11} q^{3} r^{4}+c_{13} q^{3} r^{4}-12 c_{12} q^{2} r^{5}+6 c_{15} q^{4} q_{x} \\
& +27 c_{2} q^{4} q_{x}+6 c_{5} q_{x} r^{4}+2 c_{8} r^{4} q_{x}+36 c_{1} q q_{x}^{2}+12 c_{1} q r_{x}^{2}-4 c_{8} q_{x}^{2} r-4 c_{16} r_{x}^{2} r \\
& \left.+24 q_{x} r r_{x} c_{4}\right) q_{x x}+16 c_{11} q^{3} q_{x} r^{3} r_{x}+16 c_{13} q^{3} q_{x} r^{3} r_{x}+15 c_{9} q^{2} q_{x}^{2} r r_{x}+15 c_{9} q q_{x} r^{2} r_{x}^{2} \\
& +2 c_{11} q q_{x} r^{5} r_{x}+2 c_{13} q^{5} q_{x} r r_{x}+4 c_{10} q^{2} q_{x}^{2} r r_{x}+12 c_{16} q_{x} r^{2} r_{x}^{2}+4 c_{8} q q_{x} r^{2} r_{x}^{2} \\
& +12 c_{15} q^{2} q_{x}^{2} r r_{x}+2 c_{10} q q_{x} r^{2} r_{x}^{2}+2 c_{8} q^{2} q_{x}^{2} r r_{x}+24 c_{6} r^{9} r_{x}+24 c_{3} q^{9} q_{x}+6 c_{5} r^{3} r_{x}^{3} \\
& +6 c_{2} q^{3} q_{x}^{3}+18 c_{16} r^{3} r_{x}^{3}+18 c_{15} q^{3} q_{x}^{3}+19 c_{13} q^{4} q_{x}^{2} r^{2}+2 c_{13} q^{4} r^{2} r_{x}^{2}+8 c_{6} q^{2} r^{7} r_{x} \\
& +16 c_{6} q q_{x} r^{8} c_{6}+16 c_{3} q^{8} r r_{x}+8 c_{3} q^{7} q_{x} r^{2}+6 c_{5} q_{x}^{2} r^{3} r_{x}+6 c_{2} q^{3} q_{x} r_{x}^{2}+14 c_{10} q^{3} q_{x} r_{x}^{2} \\
& +16 c_{10} q^{2} r r_{x}^{3}+6 c_{12} q^{4} r^{5} r_{x}+18 c_{12} q^{3} q_{x} r^{6}+22 c_{12} q^{2} r^{7} r_{x}+2 c_{9} q^{2} r r_{x}^{3}+c_{9} q_{x}^{2} r^{3} r_{x} \\
& +c_{9} q^{3} q_{x} r_{x}^{2}+2 c_{9} q q_{x}^{3} r^{2}+3 c_{13} q^{2} q_{x}^{2} r^{4}+3 c_{13} q^{2} q_{x}^{2} r^{4}+3 c_{11} q^{4} r^{2} r_{x}^{2}+4 c_{14} q^{6} r^{3} r_{x} \\
& +20 c_{14} q^{4} r^{5} r_{x}+4 c_{16} q_{x}^{2} r^{3} r_{x}+2 c_{16} q^{2} r r_{x}^{3}+16 c_{8} q q_{x}^{3} r^{2}+14 c_{8} q_{x}^{2} r^{3} r_{x}+4 c_{15} q^{3} q_{x} r_{x}^{2} \\
& +22 c_{7} q^{7} q_{x} r^{2}+18 c_{7} q^{6} r^{3} r_{x}+6 c_{7} q^{5} q_{x} r^{4}+4 c_{14} q^{3} q_{x} r^{6}+2 c_{15} q q_{x}^{3} r^{2}+2 c_{7} q^{8} r r_{x} \\
& +2 c_{11} q^{2} q_{x}^{2} r^{4}+19 c_{11} q^{2} r^{4} r_{x}^{2}-2 c_{4} q_{x x x x x} r+2 c_{1} q r_{x x x x x} \text {. }
\end{aligned}
$$

Solving the following equations by Maple,

$$
\frac{\delta E}{\delta q}=0, \quad \frac{\delta E}{\delta r}=0,
$$

gives $c_{3}=c_{6}=c_{7}=c_{8}=c_{9}=c_{10}=c_{11}=c_{12}=c_{13}=c_{14}=0, c_{16}=3 c_{5}, c_{15}=3 c_{2}$, and $c_{1}, c_{2}, c_{4}$, $c_{5}$ are arbitrary constants. Thus, we obtain the conserved densities, except for $q, r$,

$$
\begin{aligned}
& \rho_{1}=c_{1} q q_{x x x}+c_{4} r r_{x x x}, \\
& \rho_{2}=c_{5} r^{3} r_{x x}+3 c_{5} r^{2} r_{x}^{2}, \\
& \rho_{3}=c_{2} q^{3} q_{x x}+3 c_{2} q^{2} q_{x}^{2},
\end{aligned}
$$


where $\frac{\delta}{\delta u}=\frac{\partial}{\partial u}-D_{x} \frac{\partial}{\partial u_{x}}+D_{x}^{2} \frac{\partial}{\partial u_{x x}}-\cdots$. As for rank $R=5,6, \ldots$, we can analogously calculate the resulting conserved densities of the heat integrable system Eq. (18).

\section{Conclusion and discussion}

In the paper, we expanded the Guo hierarchy with the help of enlarged loop algebras. As a result, we obtained the isospectral and nonisospectral expanding integrable models, which reduced the linear heat equations and a type of generalized Camassa-Holm equation which was once obtained by Chang, $\mathrm{Hu}$ and $\mathrm{Li}$. By converting the usual Lax pair of the Guo hierarchy into the Lax pair in Riccati forms, the Bäcklund transformation of the Guo hierarchy was singled out. In addition, we investigated the format of conserved densities of a reduced integrable nonlinear system when $\operatorname{rank} R=4$, from which one could deduce other conserved densities when $\operatorname{rank} R=5,6, \ldots$. This approach can extend to other integrable systems, which indicates it has extensively applicable in integrable system theory. Arqub $[32,33]$ applied the generalized Taylor series formula in the Caputo sense to present the solution of the time-fractional Schrödinger equation. He also considered the solutions of systems of first-order, two-point boundary value problems for ODEs. In Ref. [34], he presented a kernel algorithm for obtaining the numerical solutions of fractional-order systems of Dirichlet function types. Meanwhile, Riza et al. [35, 36] discussed the heat and mass transfer for MHD Oldroyd-B fluid and Maxwell fluid in view of local and nonlocal differential operators. In the forthcoming days, we would like to apply the generalized $\mathrm{Tu}$ scheme to further seek new nonisospectral integrable systems and some resulting properties. Besides, we also consider whether we are able to generalize the method presented in the paper to the fractional-order differential equations in terms of the ideas showed in Refs. [32-36].

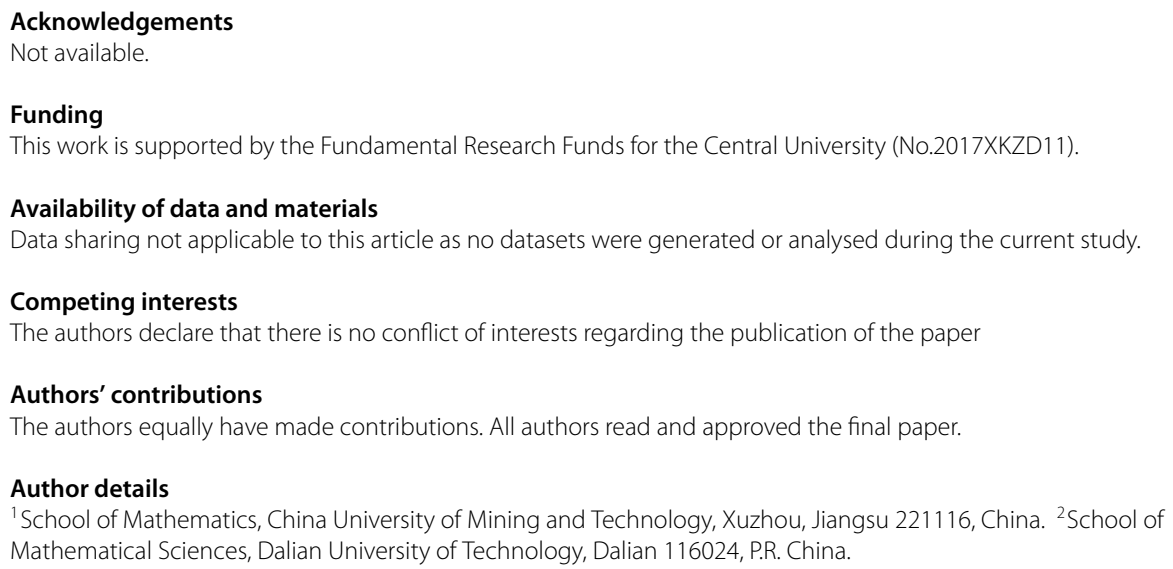

\section{Publisher's Note}

Springer Nature remains neutral with regard to jurisdictional claims in published maps and institutional affiliations.

Received: 2 May 2020 Accepted: 26 August 2020 Published online: 05 September 2020

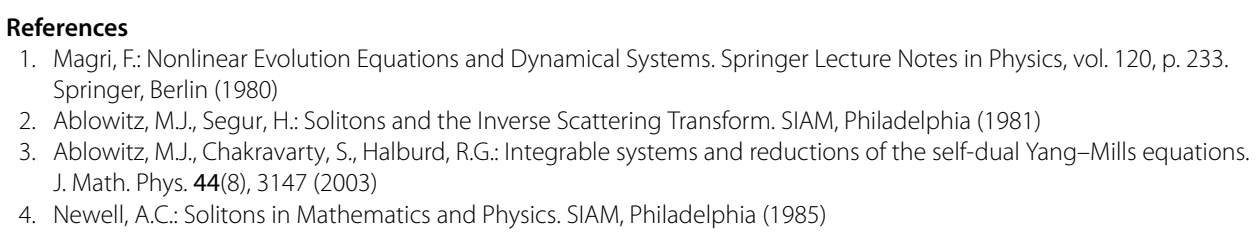


5. Tu, G.Z.: The trace identity, a powerful tool for constructing the Hamiltonian structure of integrable systems. J. Math Phys. 30(2), 330 (1989)

6. Ma, W.X.: A new hierarchy of Liouville integrable generalized Hamiltonian equations and its reduction. Chin. J. Contemp. Math. 13(1), 79 (1992)

7. Ma, W.X.: A hierarchy of Liouville integrable finite-dimensional Hamiltonian systems. Appl. Math. Mech. 13(4), 369 (1992)

8. Hu, X.B.: A powerful approach to generate new integrable systems. J. Phys. A 27, 2497 (1994)

9. Fan, E.G.: Quasi-periodic waves and asymptotic property for the asymmetrical Nizhnik-Norikov-Veselov equation. J. Phys. A 42(2), 095206 (2009)

10. Geng, X.G., Ma, W.X.: A multipotential generalization of the nonlinear diffusion equation. J. Phys. Soc. Jpn. 69(4), 985 (2000)

11. Zhang, Y.F., Zhang, H.Q.: A direct method for integrable couplings of TD hierarchy. J. Math. Phys. 43(1), 466 (2002)

12. Zhang, Y.F., Tam, H.W., Guo, F.K.: Invertible linear transformations and the Lie algebras. Commun. Nonlinear Sci. Numer. Simul. 13, 682 (2008)

13. Zhang, Y.F., Tam, H.W.: Discussion on integrable properties for higher-dimensional variable-coefficient nonlinear partial differential equations. J. Math. Phys. 54, 013516 (2013)

14. Zhang, Y.F., Tam, H.W.: Generation of nonlinear evolution equations by reductions of the self-dual Yang-Mills equations. Commun. Theor. Phys. 61, 203 (2014)

15. Qiao, Z.J.: Algebraic structure of the operator related to stationary systems. Phys. Lett. A 206, 347 (1995)

16. Guo, F.K.: Subalgebras of the loop algebra $\tilde{A}_{1}$ and integrable Hamiltonian hierarchies of equations. Acta Math. Sci. 19 , 507 (1999)

17. Ma, W.X.: An approach for constructing nonisospectral hierarchies of evolution equations. J. Phys. A, Math. Gen. 25, L719 (1992)

18. Ma, W.X.: Lax representations and Lax operator algebras of isospectral and nonisospectral hierarchies of evolution equations. J. Math. Phys. 33, 2464 (1992)

19. Qiao, Z.J.: Algebraic structure of the operator related to stationary systems. Phys. Lett. A 206, 347 (1995)

20. Qiao, Z.J.: New hierarchies of isospectral and non-isospectral integrable NLEEs derived from the Harry-Dym spectral problem. Physica A 252, 377 (1998)

21. Li, Y.S.: Symmetries of a evolution equation with the variable spectral parameters. Chin. Sci. Bull. 19, 1449 (1986) (in (hinese)

22. Li, Y.S., Zhuang, D.W.: Nonlinear evolution equations related to characteristic problems dependent on potential energy. Acta Math. Sin. 25(4), 464 (1982) (in Chinese)

23. Zhang, Y.F., Mei, J.Q., Guan, H.Y.: A method for generating isospectral and nonisospectral hierarchies of equations as well as symmetries. J. Geom. Phys. 147, 103538 (2020)

24. Zhang, Y.F., Fan, E.G., Tam, H.W.: A few expanding Lie algebras of the Lie algebra A1 and applications. Phys. Lett. A 359, $471(2006)$

25. Chang, X., Hu, X.B., Li, S.: Moment modification, multipeakons, and nonisospectral generalizations. J. Differ. Equ. 265(9), $3858(2018)$

26. Zhang, L., Qiao, Z.J.: The periodic Cauchy problem for a two-component non-isospectral cubic Camassa-Holm system. J. Differ. Equ., to be published

27. Craddock, M., Lennox, K.A.: Lie group symmetries as integral transforms of fundamental solutions. J. Differ. Equ. 232, $652(2007)$

28. Craddock, M., Platen, F.: Symmetry group methods for fundamental solutions. J. Differ. Equ. 207, 285 (2004)

29. Gui, G., Liu, Y., Olver, P.J., Qu, C.Z.: Wave-breaking and peakons for a modified Camassa-Holm equation. Commun. Math. Phys. 319, 731 (2013)

30. Tan, C., Zhang, Y.J.: Bäcklund transformations for the isospectral and nonisospectral AKNS hierarchies. J. Math. Phys. 31(9), 2150 (1990)

31. Göktas, U., Hereman, W.: Symbolic computation of conserved densities for systems of nonlinear evolution equations. J. Symb. Comput. 11, 1 (2008)

32. Arqub, O.A.: Application of residual power series method for the solution of time-fractional Schrödinger equations in one-dimensional space. Fundam. Inform. 166, 87-110 (2019)

33. Arqub, O.A.: Numerical solutions of systems of first-order, two-point BVPs based on the reproducing kernel algorithm. Calcolo 55, 31 (2018)

34. Arqub, O.A.: Numerical algorithm for solutions of fractional order systems of Dirichlet function types with comparative analysis. Fundam. Inform. 166, 111-137 (2019)

35. Riaz, M.B., Atangana, A., Abdeljawad, T.: Local and nonlocal differential operators: a comparative study of heat and mass transfer in MHD Oldroyd-B fluid with ramped wall temperature. Fractals, accepted. https://doi.org/10.1142/S0218348X20400332

36. Riaz, M.B., Atangana, A., Iftikhar, N.: Heat and mass transfer in Maxwell fluid in view of local and non-local differential operators. J. Therm. Anal. Calorim. (2020). Published online: 18 Feb. 2020. https://doi.org/10.1007/s10973-020-09383-7 